RESCEU-3/04

LBNL-54540

UT04-03

\title{
Product-Group Unification in Type IIB String Theory
}

\author{
Taizan Watari ${ }^{a}$ and T. Yanagida ${ }^{b}$ \\ a Department of Physics, University of California at Berkeley, \\ Berkeley, CA 94720, USA \\ ${ }^{b}$ Department of Physics, University of Tokyo, \\ Tokyo 113-0033, Japan
}

\begin{abstract}
The product-group unification is a model of unified theories, in which masslessness of the two Higgs doublets and absence of dimension-five proton decay are guaranteed by a symmetry. It is based on $\mathrm{SU}(5) \times \mathrm{U}(N)(N=2,3)$ gauge group. It is known that various features of the model are explained naturally, when it is embedded in a brane world. This article describes an idea of how to accommodate all the particles of the model in Type IIB brane world. The GUT-breaking sector is realized by a D3-D7 system, and chiral quarks and leptons arise from intersection of D7-branes. The D-brane configuration can be a geometric realization of the non-parallel family structure of quarks and leptons, an idea proposed to explain the large mixing angles observed in the neutrino oscillation. The tri-linear interaction of the next-to-minimal supersymmetric standard model is obtained naturally in some cases.
\end{abstract}




\section{Introduction}

Supersymmetric grand unified theories (SUSY GUTs) have been considered as the most attractive framework beyond the standard model, ever since the gauge-coupling unification was observed [1. However, the GUT scale $\left(\sim 10^{16} \mathrm{GeV}\right)$ is much higher than the energy frontier, and only a little has been known about how the theory should be.

The two following clues are the most important among all. First, the two Higgs doublets of the minimal SUSY standard model (MSSM) should be almost massless, although their mass term is not forbidden by the gauge group of the standard model. Second, dimension-five operators contributing to proton decay [2] should be sufficiently suppressed [3, 4], although they are not forbidden either. These two clues are not independent. Suppose that either of those operators is forbidden by a symmetry, and then the other is also forbidden;

$$
W \not H H \quad W \not \psi \psi \psi \psi \psi,
$$

provided the Yukawa coupling of quarks and leptons $(\psi)$ with the Higgs particles $(H)$

$$
W \ni \psi \psi H
$$

is allowed by the symmetry. Thus, the two clues can be considered as manifestation of an unbroken symmetry.

In the presence of such a symmetry, only three types of mass matrices are possible for the Higgs particles. One is the missing VEV (vacuum expectation value) type [5]. Models with the unbroken symmetry were obtained in [6, 7, 8, 9]. Another is the missing partner type [10]. Models were obtained in [11, 12, 13. The last type involves infinite number of Higgs particles, which can be obtained as a Kaluza-Klein tower [14, 15, 16].

We investigate the model [11, 12, 13, of the missing partner type. It was pointed out in [17] that various features of the model are naturally explained when it is embedded in a brane world. Thus, Ref. [18, 19] tried to embed the D $=4$ model into toroidal orientifolds of the Type IIB theory, although the variety of toroidal orientifolds was not enough to accommodate all the matter contents of the model, i.e., the particles in the GUT-symmetrybreaking sector, Higgs multiplets, and quarks and leptons. One of the purposes of this article is to illustrate an idea of how to embed all the particles in the Type IIB string theory. We do not restrict ourselves to toroidal orientifolds, and consider generic (orientifolded) Calabi-Yau 3 -folds. Various properties of the $\mathrm{D}=4$ model are translated into local properties of D-brane configuration and geometry of the Calabi-Yau 3-folds [20]. We also extract some implications to phenomenology, although an explicit string model is not obtained in this article. 
This article is organized as follows. We briefly review the $\mathrm{D}=4$ model [11, 12, 13] in section 2, and explain the motivations to embed it in a brane world. The model is embedded in the Type IIB theory in section [3. Subsection 3.1] describes the embedding of the sector responsible for breaking the unified symmetry. Subsection 3.2 is devoted to Higgs particles, where we also see that the next-to-minimal SUSY standard model (NMSSM) 21] is obtained as a special case. Subsection 3.3 describes the idea of how to obtain quarks and leptons. The origin of the $B-L$ symmetry and right-handed neutrinos are also discussed. The Dbrane configuration there provides geometric realization of the non-parallel family structure [22, 23, 24], which is suggested by the large mixing angles in the neutrino oscillation. The final section is devoted to summary and open questions.

Note: Errors in the expressions of Ramond-Ramond charge are corrected ${ }^{1}$ and two paragraphs are newly added (one in section 3.3.2 and the other in the appendix B) in version 2, in March, 2006.

\section{Brief Review of Previous Results}

\subsection{Review of the Model}

Product-group unification models based on product gauge groups, $\mathrm{SU}(5)_{\mathrm{GUT}} \times \mathrm{U}(3)_{\mathrm{H}}$ and $\mathrm{SU}(5)_{\mathrm{GUT}} \times \mathrm{U}(2)_{\mathrm{H}}$, are constructed in a four-dimensional $(\mathrm{D}=4)$ spacetime with $\mathcal{N}=1$ SUSY [11, 12, 13. They are quite similar. Thus, we only review the $\mathrm{SU}(5)_{\mathrm{GUT}} \times \mathrm{U}(3)_{\mathrm{H}}$ model in this section. For a review of the $\mathrm{SU}(5)_{\mathrm{GUT}} \times \mathrm{U}(2)_{\mathrm{H}}$ model, see [19, 28,

The "unified gauge symmetry" (or which we refer to as the GUT symmetry in the following) of the model is $\mathrm{SU}(5)_{\mathrm{GUT}} \times \mathrm{U}(3)_{\mathrm{H}}$ instead of the simple $\mathrm{SU}(5)$. Quarks and leptons are singlets of the $\mathrm{U}(3)_{\mathrm{H}}$ gauge group and form three families of $\mathbf{5}^{*}+\mathbf{1 0}$ representations of $\mathrm{SU}(5)_{\mathrm{GUT}}$. The Higgs multiplets that contain the two Higgs doublets are $H(\mathbf{5})^{i}$ and $\bar{H}\left(\mathbf{5}^{*}\right)_{i}$, which are also singlets of $\mathrm{U}(3)_{\mathrm{H}}$. Fields introduced to break the GUT symmetry are the followings: $X_{\beta}^{\alpha}(\alpha, \beta=1,2,3)$ transforming as $(\mathbf{1}, \mathbf{a d j} .=8+\mathbf{1})$ under the $\mathrm{SU}(5)_{\mathrm{GUT}} \times \mathrm{U}(3)_{\mathrm{H}}$ gauge group, and $Q_{i}^{\alpha}(i=1, \ldots, 5)+Q_{6}^{\alpha}$ and $\bar{Q}_{\alpha}^{i}(i=1, \ldots, 5)+\bar{Q}_{\alpha}^{6}$ transforming as $\left(\mathbf{5}^{*}+\mathbf{1}, \mathbf{3}\right)$ and $\left(\mathbf{5}+\mathbf{1}, \mathbf{3}^{*}\right)$. The $\mathrm{SU}(5)_{\mathrm{GUT}}$ indices are denoted by $i, j$ and those of $\mathrm{U}(3)_{\mathrm{H}}$ by $\alpha, \beta$. The chiral superfield $X_{\beta}^{\alpha}$ is also written as $X^{c}\left(t_{c}\right)_{\beta}^{\alpha}(c=0,1, \ldots, 8)$, where $t_{a}(a=1, \ldots, 8)$ are Gell-Mann matrices of the $\mathrm{SU}(3)_{\mathrm{H}}$ gauge group ${ }^{2}$ and $t_{0} \equiv \mathbf{1}_{3 \times 3} / \sqrt{6}$, where $\mathrm{U}(3)_{\mathrm{H}} \simeq \mathrm{SU}(3)_{\mathrm{H}} \times \mathrm{U}(1)_{\mathrm{H}}$.

\footnotetext{
${ }^{1}$ We thank Kentaro Hori for useful comments.

${ }^{2}$ The normalization condition $\operatorname{tr}\left(t_{a} t_{b}\right)=\delta_{a b} / 2$ is understood. Note that the normalization of the following $t_{0}$ is determined so that it also satisfies $\operatorname{tr}\left(t_{0} t_{0}\right)=1 / 2$.
} 
Table 1: $R$ charge assignment of the $\mathrm{SU}(5)_{\mathrm{GUT}} \times \mathrm{U}(3)_{\mathrm{H}}$ model

\begin{tabular}{c|ccccccc} 
Fields & $\mathbf{5}_{i}^{*}, \mathbf{1 0}^{i j}$ & $H^{i}$ & $\bar{H}_{i}$ & $X_{\beta}^{\alpha}$ & $Q_{i}^{\alpha}, \bar{Q}_{\alpha}^{i}$ & $Q_{6}^{\alpha}$ & $\bar{Q}_{\alpha}^{6}$ \\
\hline$R$ charge $(\bmod 4)$ & 1 & 0 & 0 & 2 & 0 & 2 & -2
\end{tabular}

The superpotential of the model is given [13] by

$$
\begin{aligned}
W= & \sqrt{2} \lambda_{3 \mathrm{H}} \bar{Q}^{i}{ }_{\alpha} X^{a}\left(t_{a}\right)_{\beta}^{\alpha} Q_{i}^{\beta}+\sqrt{2} \lambda_{3 \mathrm{H}}^{\prime} \bar{Q}_{\alpha}^{6} X^{a}\left(t_{a}\right)_{\beta}^{\alpha} Q_{6}^{\beta} \\
& +\sqrt{2} \lambda_{1 \mathrm{H}} \bar{Q}_{\alpha}^{i} X^{0}\left(t_{0}\right)_{\beta}^{\alpha} Q_{i}^{\beta}+\sqrt{2} \lambda_{1 \mathrm{H}}^{\prime} \bar{Q}_{\alpha}^{6} X^{0}\left(t_{0}\right)_{\beta}^{\alpha} Q_{6}^{\beta} \\
& -\sqrt{2} \lambda_{1 \mathrm{H}} v^{2} X_{\alpha}^{\alpha} \\
& +h^{\prime} \bar{H}_{i} \bar{Q}^{i}{ }_{\alpha} Q^{\alpha}{ }_{6}+h \bar{Q}_{\alpha}^{6} Q_{i}^{\alpha} H^{i} \\
& +y_{\mathbf{1 0}} \mathbf{1 0} \cdot \mathbf{1 0} \cdot H+y_{\mathbf{5}^{*}} \mathbf{5}^{*} \cdot \mathbf{1 0} \cdot \bar{H}+\cdots
\end{aligned}
$$

where the parameter $v$ is taken to be of the order of the GUT scale, $y_{10}$ and $y_{5^{*}}$ are Yukawa coupling constants for the quarks and leptons, and $\lambda_{3 \mathrm{H}}, \lambda_{3 \mathrm{H}}^{\prime}, \lambda_{1 \mathrm{H}}, \lambda_{1 \mathrm{H}}^{\prime}, h^{\prime}$ and $h$ are dimensionless coupling constants. Ellipses stand for mass terms of the neutrinos and (other) nonrenormalizable terms. The fields $Q_{i}^{\alpha}$ and $\bar{Q}_{\alpha}^{i}$ acquire vacuum expectation values (VEV's), $\left\langle Q^{\alpha}{ }_{i}\right\rangle=v \delta^{\alpha}{ }_{i}$ and $\left\langle\bar{Q}^{i}{ }_{\alpha}\right\rangle=v \delta^{i}{ }_{\alpha}$, because of the second and third lines in (3). Thus, the gauge group $\mathrm{SU}(5)_{\mathrm{GUT}} \times \mathrm{U}(3)_{\mathrm{H}}$ is broken down to that of the standard model. Mass terms of the coloured Higgs multiplets arise from the fourth line in (3) in the GUT-breaking vacuum. On the other hand, mass terms of the Higgs doublets are absent in the superpotential (3), and hence they are massless. No unwanted particle remains in the massless spectrum after the GUT symmetry is broken down to $\mathrm{SU}(3)_{C} \times \mathrm{SU}(2)_{L} \times \mathrm{U}(1)_{Y}$.

The superpotential (3) has a $(\bmod 4)-R$ symmetry; the $R$ charges $(\bmod 4)$ of the fields are given in Table 1. This symmetry forbids various dangerous operators such as mass terms of the Higgs doublets $W \supset \bar{H}_{i} H^{i}$ and proton-decay operators of dimension-four $W \supset \mathbf{5}^{*} \cdot \mathbf{1 0} \cdot \mathbf{5}^{*}$ and dimension-five $W \supset \mathbf{5}^{*} \cdot \mathbf{1 0} \cdot \mathbf{1 0} \cdot \mathbf{1 0}$. It is not broken even after the GUT symmetry is broken, because the $\operatorname{VEV}\langle\bar{Q} Q\rangle$ does not carry the $R$ charge. It is broken down to the $R$ parity when the SUSY is broken. Therefore, the two Higgs doublets have a mass term (so called $\mu$-term) only after the SUSY is broken. The dimension-five proton-decay operators have a suppression factor $\left(m_{\mathrm{SUSY}} / M_{*}\right)$, and hence they are irrelevant to the proton decay. Thus, the two difficulties in SUSY GUTs discussed in the introduction are naturally solved by the (mod 4$)-R$ symmetry

It is also remarkable [26] that the discrete $R$ symmetry is free from a mixed anomaly 
$(R \bmod 4)\left[\mathrm{SU}(3)_{\mathrm{H}}\right]^{2}$, and can be free from another mixed anomaly $(R \bmod 4)\left[\mathrm{SU}(5)_{\mathrm{GUT}}\right]^{2}$. Therefore, the symmetry can be an unbroken subgroup of a gauge symmetry. This fact sheds a light on a question why the $(\bmod 4)-R$ symmetry is preserved in an accuracy better than $10^{-14}$.

The fine structure constants of the MSSM are given at the tree level by

$$
\begin{aligned}
& \frac{1}{\alpha_{3}} \equiv \frac{1}{\alpha_{C}}=\frac{1}{\alpha_{\mathrm{GUT}}}+\frac{1}{\alpha_{3 \mathrm{H}}}, \\
& \frac{1}{\alpha_{2}} \equiv \frac{1}{\alpha_{L}}=\frac{1}{\alpha_{\mathrm{GUT}}},
\end{aligned}
$$

and

$$
\frac{1}{\alpha_{1}} \equiv \frac{\frac{3}{5}}{\alpha_{Y}}=\frac{1}{\alpha_{\mathrm{GUT}}}+\frac{\frac{2}{5}}{\alpha_{1 \mathrm{H}}},
$$

where $\alpha_{\mathrm{GUT}}, \alpha_{3 \mathrm{H}}$ and $\alpha_{1 \mathrm{H}}$ are the fine structure constants of $\mathrm{SU}(5)_{\mathrm{GUT}}, \mathrm{SU}(3)_{\mathrm{H}}$ and $\mathrm{U}(1)_{\mathrm{H}}$, respectively. Thus, the MSSM coupling constants $\alpha_{3}, \alpha_{2}$ and $\alpha_{1}$ are unified approximately, when $\alpha_{3 \mathrm{H}}$ and $\alpha_{1 \mathrm{H}}$ are sufficiently large. Although the unification is no longer a generic prediction of the present model, it is a consequence of the relatively large gauge coupling constants $\alpha_{3 \mathrm{H}}$ and $\alpha_{1 \mathrm{H}}$, or equivalently, of the relatively small constant $\alpha_{\mathrm{GUT}}$. The condition for the approximate unification is

$$
\frac{1}{\alpha_{\mathrm{GUT}}}>(10-100) \times\left(\frac{1}{\alpha_{3 \mathrm{H}}}, \frac{1}{\alpha_{1 \mathrm{H}}}\right)
$$

Now we have two remarks on the present model. First, let us neglect the weak coupling $\mathrm{SU}(5)_{\text {GUT }}$ interactions, and then the first three lines of the superpotential (3) preserves $\mathcal{N}$ $=2$ SUSY. Indeed, the chiral multiplet $X_{\beta}^{\alpha}$ is identified with the $\mathcal{N}=2$ SUSY partner of the $\mathrm{U}(3)_{\mathrm{H}}$ vector multiplet, and the chiral multiplets $Q+\bar{Q}$ are regarded as $\mathcal{N}=2$ hypermultiplets. The first two lines in the superpotential are nothing but a part of gauge interactions of the $\mathcal{N}=2$ SUSY theory when

$$
\alpha_{3 \mathrm{H}} \simeq \alpha_{3 \mathrm{H}}^{\lambda^{\left({ }^{\prime}\right)}} \equiv \frac{\lambda_{3 \mathrm{H}}^{\left({ }^{\prime}\right) 2}}{4 \pi}, \text { and } \alpha_{1 \mathrm{H}} \simeq \alpha_{1 \mathrm{H}}^{\lambda^{\left({ }^{\prime}\right)}} \equiv \frac{\lambda_{1 \mathrm{H}}^{\left({ }^{\prime}\right) 2}}{4 \pi},
$$

are satisfied. The third line is interpreted as the Fayet-Iliopoulos F-term. Now, this partial $\mathcal{N}=2$ SUSY is not only theoretically interesting, but also plays important roles in phenomenology: (i) the renormalization-group flow of $\alpha_{3 \mathrm{H}}$ is stabilized only when the $\mathcal{N}=2$ SUSY relation (88) is preserved approximately, and (ii) large threshold corrections from the 
particles in the $\mathrm{SU}(3)_{C}$-adj. representation vanish when it is preserved. For more details, see [27, 28].

The second remark is that the cut-off scale $M_{*}$ should be lower than the Planck scale $M_{\mathrm{pl}} \simeq 2.4 \times 10^{18} \mathrm{GeV}$. There are two reasons for this. First, the coupling constant $\alpha_{1 \mathrm{H}}$ becomes too large below the Planck scale because the $\mathrm{U}(1)_{\mathrm{H}}$ interaction is asymptotically non-free (see [27, 28, for more details). The second reason is that $\langle\bar{Q} Q\rangle / M_{*}^{2}$, which breaks the SU(5) GUT symmetry, should not be too small, or otherwise, it would be unable to account for the difference between the Yukawa coupling constants of the strange quark and of the muon at the unification scale.

\subsection{Motivations to Embed the Model into String Theory}

References [17, 18, 19] tried to embed the $\mathrm{D}=4$ models in subsection 2.1 into a brane world. This subsection briefly explains the motivations of the embedding. One may skip this subsection, because the construction in string theory begins in the next section.

The product-group unification model in the previous subsection preserves $\mathcal{N}=2$ SUSY in the GUT-breaking sector. However, the full theory has only $\mathcal{N}=1$ SUSY. Thus, it is a logical possibility that the multiplets $\mathcal{W}$ and $X_{\beta}^{\alpha}$ are not related by anything like $\mathcal{N}=$ 2 SUSY in their origin, neither are $Q$ and $\bar{Q}$, and that their interactions look like those of $\mathcal{N}=2$ gauge theories accidentally. However, there are two important observations against this possibility. First, the GUT-breaking sector (with the $\mathcal{N}=2$ SUSY) couples only to the $\mathrm{SU}(5)_{\text {GUT }}$ gauge fields and to the Higgs multiplets. There is no direct coupling between the sector and the chiral quarks and leptons. Thus, the GUT-breaking sector is decoupled from other sectors (with only $\mathcal{N}=1 \mathrm{SUSY}$ ) when only a few relatively weak couplings are turned off, and then, "symmetry of the sector" is well-defined. Second, the $\mathcal{N}=2$ SUSY in the GUT-breaking sector plays important roles in phenomenology [27, 28]. Therefore, the

apparent $\mathcal{N}=2$ SUSY in the sector can be a symmetry of more fundamental theory, rather than an accidental symmetry.

It is not easy to understand the coexistence of the $\mathcal{N}=2$ and $\mathcal{N}=1$ SUSY in $\mathrm{D}=$ 4 theories, but easy in theories based on higher-dimensional spacetime. Higher-dimensional theories have extended SUSY. They are compactified on curved manifolds so that D $=4$ theories with only $\mathcal{N}=1$ SUSY are obtained at low energies. Let us assume that there is a point in the internal manifold around which an extended SUSY such as $\mathcal{N}=2$ is preserved, while the full geometry has only $\mathcal{N}=1$ SUSY. If the GUT-breaking sector is localized at such an $\mathcal{N}=2$ preserving area, then, the particle contents and interactions between them 
satisfy the $\mathcal{N}=2$ SUSY, as in the model. This is the primary motivation to embed the product-group unification model into a brane-world; the GUT-breaking sector are supposed to be localized on "branes".

In string theories, $N$ coincident D-branes support $\mathrm{U}(N)$ gauge theories on their world volume. Thus, the product gauge group is quite ubiquitous in string vacua with D-branes. In particular, five coincident D-branes and $N$ coincident D-branes realize $\mathrm{U}(5)_{\mathrm{GUT}} \times \mathrm{U}(N)_{\mathrm{H}}$ gauge group ${ }^{3}(N=2,3)$. Massless fields in the bi-fundamental representations appear on the common locus of the two stacks of D-branes. References [17, 18, 19] consider that the $\mathrm{U}(N)_{\mathrm{H}}$-charged particles, i.e., the GUT-breaking sector, is obtained on a world volume of D3-branes, and that the $\mathrm{SU}(5)_{\text {GUT }}$ gauge field comes from D7-branes. Rotational symmetry of the internal space plays the role of the $R$ symmetry.

When the total volume of the internal space is large, the fundamental scale $M_{*}$ is lower than the Planck scale $M_{\mathrm{pl}}$. This is one of the features required for the $\mathrm{SU}(5)_{\mathrm{GUT}} \times \mathrm{U}(N)_{\mathrm{H}}$ model $(N=2,3)$. Moreover, the world volume of the $\mathrm{D}$-branes for the $\mathrm{U}(5)_{\text {GUT }}$ gauge group can also be large since the total volume is large. When the world volume is moderately large, i.e., $10-100$ in units of $1 / M_{*}$, the fine structure constants of the $\mathrm{U}(5)_{\text {GUT }}$ interactions are relatively small by $1 / 10-1 / 100$ when compared with those of $\mathrm{U}(3)_{\mathrm{H}}$. This is also a desired feature of the model.

In summary, the string vacua with D-branes may be able to explain five features ${ }^{4}$ of the $\mathrm{SU}(5)_{\mathrm{GUT}} \times \mathrm{U}(N)_{\mathrm{H}}$ model $(N=2,3)$, namely, (i) the partial $\mathcal{N}=2 \mathrm{SUSY}$ in the GUTbreaking sector, (ii) the origin of the product gauge group, (iii) the origin of the $R$ symmetry, (iv) the cut-off scale lower than the Planck scale and (v) the relatively small coupling constant of the $\mathrm{SU}(5)_{\text {GUT }}$ interactions. Therefore, we consider that it is well motivated to embed the model into string vacua.

\footnotetext{
${ }^{3}$ The extra $\mathrm{U}(1)$ gauge symmetry contained in $\mathrm{U}(5)_{\mathrm{GUT}} \times \mathrm{U}(3)_{\mathrm{H}}$ can be identified with the $B-L$ symmetry. It requires right-handed neutrinos so that its triangle anomaly is cancelled. Right-handed neutrinos can lead to tiny masses of left-handed neutrinos through the see-saw mechanism [29]. See discussion in subsubsection 3.3

${ }^{4}$ It was also pointed out in 17 that the SUSY flavour problem may be solved through the gauginomediation mechanism [30 in this framework. This is because the $\mathrm{SU}(5)_{\text {GUT }}$ gauge field propagates in the internal dimensions, say, on the D7-branes, while quarks and leptons may be further localized inside the world volume of SU(5) GUT (see subsection 3.3). After 17 was published, however, phenomenological and theoretical problems of the gaugino mediation were pointed out by [31] and [32, respectively. Further investigation is necessary on this issue [33, 34].
} 


\section{Construction in Type IIB Theory}

We consider that the $\mathrm{SU}(5)_{\mathrm{GUT}} \times \mathrm{U}(N)_{\mathrm{H}}$ gauge symmetry $(N=2,3)$ arises from space-filling D-branes. The Type IIB theory is compactified on Calabi-Yau 3-folds, and space-filling Dbranes and orientifold planes wrap holomorphic cycles [35, 36, so that $\mathrm{D}=4$ gauge theories with $\mathcal{N}=1$ SUSY are obtained at low energies. Quarks and leptons arise from open strings connecting those D-branes. This is the picture we have in mind.

Reference [20] emphasized that the discovery of D-branes brought a new method into string phenomenology. Provided that a gauge theory is obtained from D3-branes trapped at an orbifold singularity, some of (though not necessarily all of) phenomenological properties of the gauge theory are determined only from the local geometry and local configuration of the D-branes. One does not have to know the whole information of the Calabi-Yau 3fold in obtaining (some of) phenomenological predictions. References [37, 8, 38, and [39] share the same philosophy in a slightly extended form; geometry around singularities and some properties of cycles on which Kaluza-Klein monopoles and D6-branes are wrapped are sometimes sufficient information in deriving some of phenomenological consequences. This approach can be called as local construction or bottom-up approach.

This approach enables us to proceed just as particle physicists have been doing. Each sector of a phenomenological model is realized by D-branes. Only minimal requirement is imposed on the local geometry so that the desired properties of the model are obtained. After that, the local configuration of D-branes for various sectors are combined in a suitable way to form the whole model. One can leave any properties in such a construction, if one does not have to fix them. Yet, one can hope to obtain some phenomenological consequences. This is the approach we adopt in this article.

\subsection{GUT-Breaking Sector}

$N$ fractional D3-branes $(N=2,3)$ and six D7-branes are introduced for the GUT-breaking sector. The local geometry around the fractional D3-branes (the GUT-breaking sector) is $\mathbf{C}^{2} / \mathbf{Z}_{M} \times \mathbf{C}$. The fractional D3-branes are at the orbifold singularity, and the D7-branes are stretched in $\mathbf{C}^{2} / \mathbf{Z}_{M}$ direction while not in $\mathbf{C}$ direction (see Fig. (1). Then, $\mathrm{D}=4 \mathcal{N}=2$ SUSY is preserved in the field theory localized at the fractional D3-branes, due to the local D-brane configuration and geometry.

When the $N$ fractional D3-branes belong to the same representation of the orbifold group $\mathbf{Z}_{M}$, they are trapped at the orbifold singularity. We have a $\mathrm{U}(N)$ vector multiplet of $\mathcal{N}$ 


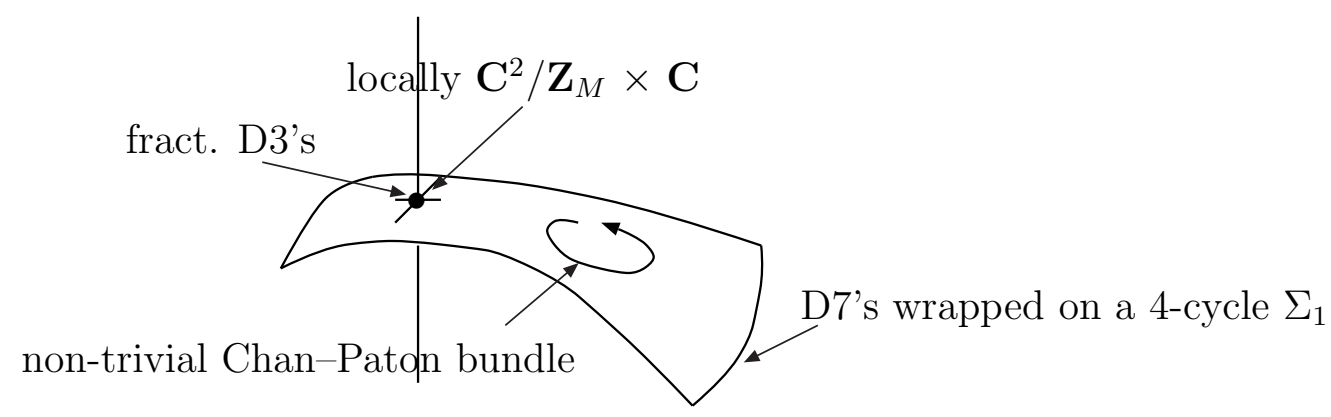

Figure 1: A schematic picture of the local geometry of $C Y_{3}$ and D-brane configuration in it. Only the parts relevant to subsection 3.1 (GUT-breaking sector) and 3.2 (Higgs particles) are described here.

$=2$ SUSY from the D3-D3 open strings, while massless hypermultiplets from the D3-D3 strings are projected out. If the six D7-branes are in the same representation of $\mathbf{Z}_{M}$ as the $N$ fractional D3-branes are, then the massless hypermultiplets from the D3-D7 open strings are not projected out from the spectrum. In summary, we have an $\mathcal{N}=2 \operatorname{SUSY} \mathrm{U}(N)$ gauge theory of $\mathrm{D}=4$ spacetime, whose matter contents are six hypermultiplets in the fundamental representation. The flavour symmetry of this hypermultiplets is $\mathrm{U}(6)$, which arises from the D7-branes. The matter content is just what we want for the GUT-breaking sector. Thus, we identify the $\mathrm{U}(N)$ vector multiplet and hypermultiplets with the $\mathrm{U}(N)_{\mathrm{H}}$ vector multiplet $\left(\mathcal{W}, X_{\beta}^{\alpha}\right)$ and the hypermultiplets $\left(Q_{k}^{\alpha}, \bar{Q}^{k}{ }_{\alpha}\right)$ in the bi-fundamental representation $\left(N, 5^{*}+\mathbf{1}\right)$ (or equivalently, in $\left(N^{*}, \mathbf{5}+\mathbf{1}\right)$ ) in the previous section.

The centre-of-mass $\mathrm{U}(1)$ part of the $\mathrm{U}(N)_{\mathrm{H}}$ vector multiplets, however, is no longer massless at the quantum level [40. Thus, there should be at least one more fractional D3-brane at the $\mathbf{C}^{2} / \mathbf{Z}_{M}$ singularity. This extra D-brane is required also because of the consistency condition associated with the $\mathbf{C}^{2} / \mathbf{Z}_{M}\langle\sigma\rangle$ singularity. The Ramond-Ramond tadpole cancellation condition [4] is given by 42 ]

$$
\operatorname{tr}\left(\gamma_{7 ; \sigma^{k}}\right)-4 \sin ^{2}\left(\frac{\pi k}{M}\right) \operatorname{tr}\left(\gamma_{3 ; \sigma^{k}}\right)=0, \quad(k=1, \ldots, M-1) .
$$

All fractional D3-branes and D7-branes, no matter where they are in the $\mathbf{C}$ direction, contribute to the condition. Since the Ramond-Ramond tadpoles do not cancel in the $\mathrm{U}(3)_{\mathrm{H}} \times$ $\mathrm{U}(6)_{D 7}$ model, extra D-branes have to be introduced. We assume that there is a D-brane configuration which is consistent with the tadpole condition, and is free from unwanted extra massless particles. Finding an explicit configuration is left to further investigation.

Even though we do not specify the D-brane configuration completely, there is still some- 
thing we can learn. The $\mathrm{U}(1)_{\mathrm{H}}$ vector field has to be massless, and hence should not couple to the twisted-sector Ramond-Ramond field. Thus, the origin of the Fayet-Iliopoulos F-term parameter in (3) is not the VEV of the twisted NS-NS sector fields, but something else ${ }^{5}$. It is also easy to see that the gauge coupling constants of $\mathrm{SU}(N)_{\mathrm{H}}$ and $\mathrm{U}(1)_{\mathrm{H}}$ do not satisfy a relation $\alpha_{N \mathrm{H}}=\alpha_{1 \mathrm{H}}$ at the string scale.

Let us now turn our attention to the six D7-branes. They are wrapped on a holomorphic 4-cycle so that the $\mathcal{N}=1$ SUSY is preserved [35, 36]. The cycle is denoted by $\Sigma_{1}$ (see Fig. (1).

Now, the flavour symmetry $\mathrm{U}(6)$ becomes dynamical. An $\mathrm{SU}(5)$ subgroup of this $\mathrm{U}(6)$ symmetry is identified with the $\mathrm{SU}(5)_{\mathrm{GUT}}$. The $\mathrm{U}(6)$ (and hence $\mathrm{SU}(5)_{\mathrm{GUT}}$ ) gauge coupling constant is given by

$$
\frac{1}{\alpha_{U(6)}}=\frac{1}{g_{s}} \frac{\operatorname{vol}\left(\Sigma_{1}\right)}{\left(2 \pi \sqrt{\alpha^{\prime}}\right)^{4}},
$$

while

$$
\frac{1}{\alpha_{N \mathrm{H}}}=\frac{1}{g_{s}} .
$$

Thus, the string coupling is determined by the value of $\alpha_{N \mathrm{H}}-g_{s} \sim 1 / 2-2$. A moderately large volume of the cycle $\Sigma_{1}$

$$
\frac{\operatorname{vol}\left(\Sigma_{1}\right)}{\left(2 \pi \sqrt{\alpha^{\prime}}\right)^{4}} \sim(10-100)
$$

leads to the relatively weak coupling of $\mathrm{SU}(5)_{\mathrm{GUT}}$ in (7), or equivalently, to the approximate unification of the MSSM gauge coupling constants. Note also that the string scale is given by

$$
\frac{1}{\sqrt{\alpha}^{\prime}}=\left(\pi g_{s}^{2} \frac{\left(2 \pi \sqrt{\alpha}^{\prime}\right)^{6}}{\operatorname{vol}\left(C Y_{3}\right)}\right)^{\frac{1}{2}} M_{\mathrm{pl}} \simeq \sqrt{\pi \alpha_{\mathrm{GUT}} \alpha_{N \mathrm{H}}}\left(\frac{\left(2 \pi \sqrt{\alpha}^{\prime}\right)^{2}}{\operatorname{vol}\left(C Y_{3}\right) / \operatorname{vol}\left(\Sigma_{1}\right)}\right)^{\frac{1}{2}} \times 2.4 \times 10^{18} \mathrm{GeV},
$$

and hence can be sufficiently low when the total volume of the Calabi-Yau 3-fold is sufficiently large. Typically, $\left(\operatorname{vol}\left(C Y_{3}\right) / \operatorname{vol}\left(\Sigma_{1}\right)\right) / 4 \pi \alpha^{\prime} \sim 100$ is necessary for $1 / \sqrt{\alpha^{\prime}} \sim 10^{17} \mathrm{GeV}$.

The moderately large volume required above guarantees that the supergravity provides a good description to some extent, i.e., the vacuum is not in a purely stringy regime. We also see from above that the Kaluza-Klein scale is roughly of the order of the GUT scale. Thus, it is tempting to speculate that the origin of the GUT scale, and hence of the Fayet-Iliopoulos term in (3), has something to do with the Kaluza-Klein scale.

If the 3-form fluxes are introduced for the moduli stabilization [43, the background 3form field strength is of the order of $\alpha^{\prime} / R^{3}$, and the 2-form potential of the order of $\alpha^{\prime} / R^{2}$

\footnotetext{
${ }^{5}$ This issue is discussed later again.
} 
[44, where $R$ is the typical length scale of the internal manifold. Then, a dimension-two quantity (for the Fayet-Iliopoulos parameter $v^{2}$ ) is obtained from the background 2-form potential by multiplying $1 / \alpha^{\prime}$, which is $1 / \alpha^{\prime} \times\left(\alpha^{\prime} / R^{2}\right) \sim 1 / R^{2}$. Thus, it may be that the B-field background for the moduli stabilization is also the origin of the Fayet-Iliopoulos term 17]. Although the above idea is too naive, and neither the moduli stabilization nor back reaction is considered, yet it is an interesting idea for the origin of the GUT scale.

The U(6) symmetry has to be reduced to SU(5) (and extra U(1)'s). To this end, nontrivial background of $\mathrm{U}(1) \subset \mathrm{SU}(6)$ field strength is introduced; $\mathrm{U}(1)$ is a subgroup of $\mathrm{SU}(6)$ that commutes with $\mathrm{SU}(5)$. Let the Chan-Paton bundles on the six D7-branes be denoted by

$$
\lambda_{(\mathbf{5} \oplus 1) \times\left(\mathbf{5}^{*} \oplus 1\right)} \rightarrow\left(\begin{array}{c|c}
E_{\mathbf{5 5}} & E_{\mathbf{5 6 *}} \\
\hline E_{6 \mathbf{5}^{*}} & E_{66^{*}}
\end{array}\right) .
$$

The vector bundles $E_{56^{*}}$ and $E_{65^{*}}$ are non-trivial ${ }^{6}$, and the spectrum no longer respects the U(6) symmetry. The background field strength has to satisfy the "generalized Hitchin equations" in [45] so that the $\mathrm{D}=4 \mathcal{N}=1$ SUSY is preserved.

The spectrum of the D7-D7 open strings preserves only $\mathcal{N}=1$ SUSY, unless the local geometry around the $\Sigma_{1}$ hypersurface satisfies special properties. We have an $\mathrm{SU}(5)$ vector multiplet of $\mathcal{N}=1$ SUSY, which is identified with the $\mathrm{SU}(5)_{\text {GUT }}$ vector multiplet. There are two U(1) symmetries coming from the D7-D7 open strings at the classical level. But, they usually have triangle anomalies, which are cancelled by the (generalized) Green-Schwarz mechanism. In this case, the vector fields of the symmetries are not massless.

The number of massless $\mathcal{N}=1$ SUSY chiral multiplets in the $\mathrm{SU}(5)_{\mathrm{GUT}}$-adj. representation is given by $h^{0}\left(T \Sigma_{1} \oplus \mathcal{N}_{\Sigma_{1}}\right)=h^{0}\left(T \Sigma_{1} \oplus K_{\Sigma_{1}}\right)$ [46, 47], where $T \Sigma_{1}, \mathcal{N}_{\Sigma_{1}}$ and $K_{\Sigma_{1}}$ are tangent, normal and canonical bundles on $\Sigma_{1}$, respectively, and $h^{0}$ stands for the number of global holomorphic sections of the corresponding vector bundles. Thus, the SU(5) GUT $^{-}$ adj. chiral multiplets are usually absent in the low-energy spectrum, just as desired in the $\mathrm{SU}(5)_{\mathrm{GUT}} \times \mathrm{U}(N)_{\mathrm{H}}$ model $(N=2,3)$. For the massless chiral multiplets from the open strings connecting five and the other D7-branes, see subsection 3.2 .

The Calabi-Yau geometry has been required so far to satisfy the following properties. It has to have a holomorphic 4-cycle, $\Sigma_{1}$, on which there is a point whose local geometry should be (approximately) $\mathbf{C}^{2} / \mathbf{Z}_{M} \times \mathbf{C}$. The volume of the Calabi-Yau 3-fold is moderately large in units of string length in the directions both tangential and transverse to the cycle $\Sigma_{1}$. The cycle has to satisfy $h^{0}\left(T \Sigma_{1} \oplus K_{\Sigma_{1}}\right)=0$. It is not hard to find a Calabi-Yau 3-fold that

\footnotetext{
${ }^{6}$ The vector bundles have to be trivial around the GUT-breaking sector, though.
} 
possesses the properties described above; one can find an example $\mathbf{T}^{6} / \mathbf{Z}_{12}$ in [18, 19].

\section{$3.2 \quad$ Higgs Multiplets}

The $\mathrm{SU}(5)_{\mathrm{GUT}} \times \mathrm{U}(3)_{\mathrm{H}}$ model requires Higgs multiplets, $H^{i}(\mathbf{5}, \mathbf{1})+\bar{H}_{i}\left(\mathbf{5}^{*}, \mathbf{1}\right)$, in the spectrum. It is economical if they are also obtained from the D7-branes wrapped on $\Sigma_{1}$. Moreover, it is desirable to obtain the Higgs multiplets in this way, because they have non-vanishing wave functions at the $\mathbf{C}^{2} / \mathbf{Z}_{M}$ singularity (i.e., the locus of the GUT-breaking sector), and hence the interactions in the fourth line of (3) are expected not to be suppressed ${ }^{7}$. Thus, we describe in this subsection how to obtain the Higgs multiplets from the open strings connecting five D7-branes and another(others) wrapping the same 4-cycle $\Sigma_{1}$.

Since one D7-brane has already been introduced in addition to the five D7-branes for $\mathrm{SU}(5)_{\mathrm{GUT}}$, let us first discuss whether it is possible to obtain both Higgs multiplets $H^{i}(\mathbf{5})$ and $\bar{H}\left(\mathbf{5}^{*}\right)$ from those six D7-branes. The massless Higgs multiplets are in the spectrum when $h^{0}\left(E_{56^{*}} \otimes\left(T \Sigma_{1} \oplus K_{\Sigma_{1}}\right)\right)=1$ and $h^{0}\left(E_{65^{*}} \otimes\left(T \Sigma_{1} \oplus K_{\Sigma_{1}}\right)\right)=1$. Then, it follows that there is a global section also in the tensor product of the two vector bundles above. This implies that

$$
h^{0}\left(\otimes^{2}\left(T \Sigma_{1} \oplus K_{\Sigma_{1}}\right)\right) \geq 1
$$

since the vector bundle $E_{56^{*}} \otimes E_{65^{*}}$ is trivial. Thus, we see that the geometry along the 4-cycle $\Sigma_{1}$ has to satisfy the extra condition (15) in order that the two Higgs quintets are obtained from the six D7-branes.

There is another possibility where the two Higgs multiplets are obtained even when the 4-cycle $\Sigma_{1}$ does not satisfy the condition (15). To this end, another D7-brane is introduced which is also wrapped on the 4-cycle $\Sigma_{1}$. Let the Chan-Paton bundles for massless open strings be denoted by

$$
\lambda_{(\mathbf{5} \oplus 1 \oplus 1) \times(\mathbf{5} \oplus 1 \oplus 1)^{*}} \rightarrow\left(\begin{array}{c|l|l}
E_{55^{*}} & E_{56^{*}} & E_{57^{*}} \\
\hline E_{65^{*}} & E_{66^{*}} & E_{67^{*}} \\
\hline E_{75^{*}} & E_{76^{*}} & E_{77^{*}}
\end{array}\right) .
$$

The condition (15) needs not be satisfied now, since, say, $E_{57^{*}} \otimes E_{65^{*}} \simeq E_{67^{*}}$ is not trivial generically.

The NMSSM is quite natural in this framework. Suppose that $h^{0}\left(E_{65^{*}} \otimes\left(T \Sigma_{1} \oplus K_{\Sigma_{1}}\right)\right)=1$

\footnotetext{
${ }^{7}$ We do not discuss how the superpotential of the low-energy $\mathrm{D}=4$ effective theory is generated.
} 
and $h^{0}\left(E_{\mathbf{5}^{*}} \otimes\left(T \Sigma_{1} \oplus K_{\Sigma_{1}}\right)\right)=1$, so that the two Higgs quintets are obtained. Then, it follows ${ }^{8}$ that $h^{0}\left(E_{67^{*}} \otimes \wedge^{2}\left(T \Sigma_{1} \oplus K_{\Sigma_{1}}\right)\right) \geq 1$. If this is due to a trivial bundle in $E_{67^{*}} \otimes \wedge^{2}\left(T \Sigma_{1} \oplus K_{\Sigma_{1}}\right)$, then its dual bundle $E_{76^{*}} \otimes\left(T \Sigma_{1} \oplus K_{\Sigma_{1}}\right)$ also has a global holomorphic section. Thus, another massless chiral multiplet is in the spectrum, which comes from the open strings connecting sixth and seventh D7-branes. This chiral multiplet is a singlet of $\mathrm{SU}(5)_{\text {GUT }}$, which is denoted by $S$. The interactions on the D7-branes predict a tri-linear coupling in the low-energy effective superpotential,

$$
W \ni S_{6}^{7} \bar{H}_{i}^{6} H_{7}^{i}
$$

which is nothing but the interaction of NMSSM [21. The coefficient of this operator is of the order of the gauge coupling constant of $\mathrm{SU}(5)_{\mathrm{GUT}}$ at the classical level. But, we do not understand all the dynamics (including non-perturbative one) that generates necessary superpotential couplings, and hence it is impossible to derive a quantitative prediction for the value of this coupling constant.

What is shown above is a generalization of what was found in [19]. There, the two Higgs quintets were obtained along with the singlet $S$ in the explicit toroidal orientifold model based on $\mathbf{T}^{6} / \mathbf{Z}_{12}$.

Notice that the existence of the singlet $S$ is due to the property $\mathcal{N}_{\Sigma_{1}} \simeq K_{\Sigma_{1}}$, which is an immediate consequence of the definition of Calabi-Yau manifolds. The NMSSM interaction (17) is the immediate consequence of the $\mathcal{N}=4$ SUSY interactions on the D7-branes. The tri-linear interaction reflects the fact that the internal manifold has three complex dimensions. Thus, it is quite interesting if the NMSSM interaction is discovered in future collider experiments.

\subsection{Quarks and Leptons}

Let us now illustrate the idea of how the quarks and leptons can be constructed in Type IIB vacua in a way consistent with the model we have constructed in the preceding subsections. The model of unified theories we have considered is based on SU(5)-symmetric matter contents, and not on $\mathrm{SO}(10)$-symmetric ones. This is the case when the five D7-branes are not wrapped on the same cycle as the O7-plane and their orientifold images are (see Fig. 2).

\footnotetext{
${ }^{8} h^{0}\left(E_{6 \mathbf{5}^{*}} \otimes E_{\mathbf{5} 7^{*}} \otimes \wedge^{2}\left(T \Sigma_{1} \oplus K_{\Sigma_{1}}\right)\right)=1$ does not follow when $E_{6 \mathbf{5}^{*}} \simeq E_{\mathbf{5} 7^{*}}$. However, we do not consider this case in this article. This is because the isomorphism implies that the Chan-Paton bundle $E_{57 *}$ is also trivial around the $\mathbf{C}^{2} / \mathbf{Z}_{M}$ singularity in subsection 3.1. U(7) symmetry is enhanced there, and another massless hypermultiplet in the $\mathrm{U}(3)_{\mathrm{H}^{-}}$(anti)fund. representation appears in the spectrum, invalidating the gauge-coupling unification.
} 


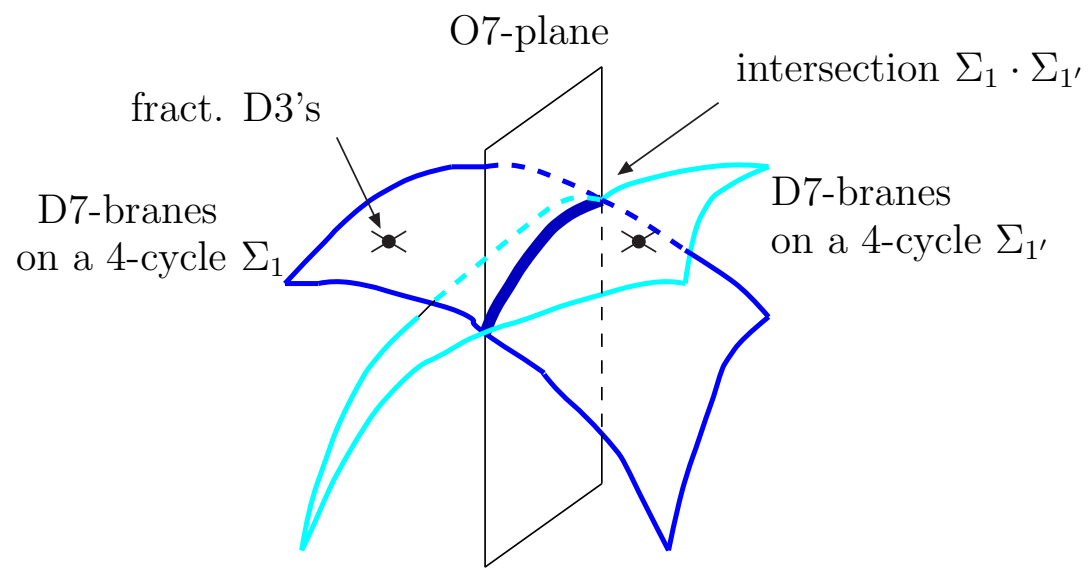

Figure 2: A schematic picture of the configuration for the chiral multiplets in the antisymmetric-tensor representation. A holomorphic 4-cycle $\Sigma_{1^{\prime}}$ is the image of $\Sigma_{1}$ under an involution associated with the O7-plane. They intersect at a holomorphic curve $\Sigma_{1} \cdot \Sigma_{1^{\prime}}$ (thick curve in the figure). D7-branes are wrapped on the 4-cycles, and the $\mathrm{SU}(5)_{\text {GUT }}$ gauge field propagates all over the world volume. The matter in the anti-symmetric-tensor representation arises at the intersection. The GUT-breaking sector is realized by fractional D3-branes located at a $\mathbf{C}^{2} / \mathbf{Z}_{2} \times \mathbf{C}$ singularity on the 4-cycle $\Sigma_{1}$.

On the other hand, a matter in the SU(5) anti-symmetric-tensor representation arises on a locus where D-branes and their orientifold images coincide. Therefore, the D7-branes for the $\mathrm{SU}(5)_{\text {GUT }}$ gauge group should intersect with their orientifold mirror images in the CalabiYau 3-fold, and the anti-symmetric-tensor representation should arise there (see Fig. 22). $\mathrm{SU}(5)_{\mathrm{GUT}} \mathbf{- 1 0}$ representation is localized on a complex curve in the Calabi-Yau 3-fold (Fig. 2).

\subsubsection{Toy Model for Chiral Matter at D7-D7 Intersection on Orbifold}

Let us first describe a simple toy model that shows the essential feature of how the chiral matter in the $\mathrm{SU}(5)_{\mathrm{GUT}} \mathbf{- 1 0}$ representation is obtained. An orbifold is used to construct the toy model, where the complex curve where the chiral matter lives is compact, while the total Calabi-Yau space is non-compact. This semi-local model is considered as a model of the local neighbourhood of the curve where the D7-branes intersect. We describe more generalized model in subsubsection 3.3.2, where we do not restrict ourselves to orbifolds, in 


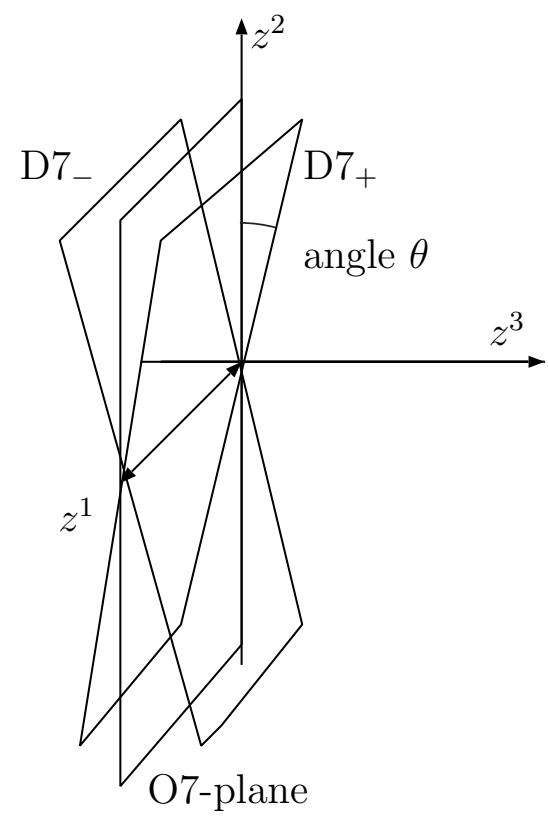

Figure 3: Toy model explained in subsubsection 3.3.1, $z^{1}$ is the coordinate of $\mathbf{T}^{2}$, and $\left(z^{2}, z^{3}\right)$ are those of $\mathbf{C}^{2}$. Orientifold projection is associated with the reflection in $z^{3}$-direction, and hence the O7-plane is the fixed locus of the reflection, i.e., $z^{3}=0$ hypersurface. D7_ is the orientifold mirror image of $\mathrm{D} 7_{+}$.

which arbitrary number of families of chiral matter can be obtained.

Let us suppose that the six internal dimensions are of the form $\mathbf{T}^{2} \times \mathbf{C}^{2}$, whose complex coordinates are $z_{1}$ for $T^{2}$ and $\left(z_{2}, z_{3}\right)$ for $\mathbf{C}^{2}$. Two stacks of space-filling D7-branes are stretched in $z_{1} \wedge \bar{z}_{1} \wedge\left(\cos \theta z_{2} \pm \sin \theta z_{3}\right) \wedge\left(\cos \theta \bar{z}_{2} \pm \sin \theta \bar{z}_{3}\right)$ (see Fig. [3). Here, $\theta$ is a constant angle and is arbitrary, unless it is an integral multiple of $\pi / 2$. These stacks are mapped with each other by an $\mathrm{SU}(2)$ transformation ${ }^{9}$. Thus, we have a $\mathrm{D}=6(1,0)$-SUSY gauge theory if the volume of $T^{2}$ is infinite, and a $\mathrm{D}=4 \mathcal{N}=2$ SUSY gauge theory if the volume is finite. When the O7-plane is stretched in the $\left(z_{1}, z_{2}\right)$ complex planes, i.e., it is wrapped on the $z_{3}=0$ hypersurface, one stack of D7-branes D7_ in Fig. 3 is the orientifold image of the other stack D7+. The gauge theory consists only of a hypermultiplet in the anti-symmetric-tensor representation.

The system described above still preserves $\mathcal{N}=2$ SUSY, and the matter contents are

\footnotetext{
${ }^{9}$ Indeed, an $\mathrm{SO}(2)$ transformation between $z_{2}$ and $z_{3}$ by angle $\mp 2 \theta$ does the job.
} 
vector-like in the $\mathrm{D}=4$ effective theory. Thus, we impose an orbifold projection to obtain a chiral theory. Let us consider a $\mathbf{Z}_{N}$ orbifold $^{10}$, where the three complex coordinates transform as

$$
z_{1} \mapsto e^{2 i \alpha} z_{1}, \quad z_{2} \mapsto e^{-i \alpha} z_{2}, \quad z_{3} \mapsto e^{-i \alpha} z_{3}
$$

This transformation belongs to $\mathrm{SU}(3)$, and hence the $\mathrm{D}=4 \mathcal{N}=1$ SUSY is preserved. Note that it also preserves the world volumes of both stacks of D7-branes, and hence extra D-branes do not have to be introduced as images of the $\mathbf{Z}_{N}$ action. When the matrix twisting the Chan-Paton indices is chosen suitably, two states among four in the NS sector and two states among four in the $\mathrm{R}$ sector survive the orbifold projection. Thus, one chiral multiplet in the anti-symmetric-tensor representation is in the massless spectrum, while its hermitian conjugate is projected out. For more details, see the appendix A.

\subsubsection{Model of Chiral Matter at D7-D7 Intersection Using Curved Manifold}

The toy model above yields an $\mathcal{N}=1$ chiral multiplet in the anti-symmetric-tensor representation, yet we have only one family; one hypermultiplet is obtained on $\mathbf{R}^{3,1} \times \mathbf{T}^{2}$, half of the massless modes (one chiral multiplet) remains in the spectrum, while the other half is projected out. The only one family, however, is not a generic feature of the chiral matters obtained at the intersection of D7-branes, if we do not restrict ourselves to the simplest model above, which uses $\mathbf{T}^{2}$ and its orbifolds.

Suppose that there is an O7-plane in a Calabi-Yau 3-fold. A stack of D7-branes are wrapped on the holomorphic 4-cycle $\Sigma_{1}$ and another stack of D7-branes are on another holomorphic 4-cycle $\Sigma_{1^{\prime}}$. The 4-cycle $\Sigma_{1^{\prime}}$ is supposed to be the image of $\Sigma_{1}$ under the involution associated with the orientifold plane. We consider that each stack consists of six or seven D7-branes, or more, if necessary. Yang-Mills fields on the D7-branes on $\Sigma_{1^{\prime}}$ are identified with those on $\Sigma_{1}$. Their Kaluza-Klein zero modes have been discussed in subsection 3.1 and 3.2. Now let us assume that the two 4-cycles $\Sigma_{1}$ and $\Sigma_{1^{\prime}}$ intersect at a holomorphic curve $\Sigma_{1} \cdot \Sigma_{1^{\prime}}$ as in the toy model of subsubsection 3.3.1 (see Fig. 2). The massless matter contents localized at the intersection consist of two complex bosons and a Weyl fermion of $\mathrm{D}=6$ spacetime. The two bosons are sections of $E_{\Sigma_{1}} \otimes E_{\Sigma_{1^{\prime}}}^{*} \otimes\left(\mathcal{N}_{\Sigma_{1} \cdot \Sigma_{1^{\prime}} \mid \Sigma_{1}} \otimes \mathcal{N}_{\Sigma_{1^{\prime}} \cdot \Sigma_{1^{\prime}} \mid \Sigma_{1^{\prime}}}\right)^{1 / 2}$ and $E_{\Sigma_{1}}^{*} \otimes E_{\Sigma_{1^{\prime}}} \otimes\left(\mathcal{N}_{\Sigma_{1} \cdot \Sigma_{1^{\prime}} \mid \Sigma_{1}} \otimes \mathcal{N}_{\Sigma_{1} \cdot \Sigma_{1^{\prime}} \mid \Sigma_{1^{\prime}}}\right)^{1 / 2}$ [46, [59, 48], and the fermion is a section of $E_{\Sigma_{1}} \otimes E_{\Sigma_{1^{\prime}}}^{*}$ [49, 59, 48]; here, $\mathcal{N}_{\Sigma_{1} \cdot \Sigma_{1^{\prime}} \mid \Sigma_{1}}$ is the normal bundle on the intersection $\Sigma_{1} \cdot \Sigma_{1^{\prime}}$ associated with the embedding $\left.\left(i_{\Sigma_{1^{\prime}}}\right)\right|_{\Sigma_{1} \cdot \Sigma_{1^{\prime}}}: \Sigma_{1} \cdot \Sigma_{1^{\prime}} \hookrightarrow \Sigma_{1}$, and $\mathcal{N}_{\Sigma_{1} \cdot \Sigma_{1^{\prime}} \mid \Sigma_{1^{\prime}}}$ with the embedding

\footnotetext{
${ }^{10}$ The $\mathbf{Z}_{N}$ has to be a symmetry of $T^{2}$, and $\alpha \in(2 \pi / N) \mathbf{Z}$.
} 
$\left.\left(i_{\Sigma_{1}}\right)\right|_{\Sigma_{1} \cdot \Sigma_{1^{\prime}}}: \Sigma_{1} \cdot \Sigma_{1^{\prime}} \hookrightarrow \Sigma_{1^{\prime}} . E_{\Sigma_{1}}$ denotes the Chan-Paton U(1) bundle on $\Sigma_{1}$ that leads to $E_{\Sigma_{1}} \otimes E_{\Sigma_{1}}^{*} \simeq E_{55^{*}} \oplus E_{56^{*}} \oplus E_{65^{*}} \oplus E_{66^{*}} \oplus \cdots$ introduced in subsection 3.1 (and 3.2). $E_{\Sigma_{1^{\prime}}}$ plays the same role on $\Sigma_{1^{\prime}}$ as $E_{\Sigma_{1}}$ does on $\Sigma_{1}$.

The complex curve $\Sigma_{1} \cdot \Sigma_{1^{\prime}}$ is compact, and hence we obtain massless modes of D = 4 theories through the Kaluza-Klein reduction of the two complex bosons and the Weyl fermion on the curve. The net number of massless complex scalar fields in chiral multiplets is given by

$$
\begin{aligned}
N_{B}= & h^{0}\left(\Sigma_{1} \cdot \Sigma_{1^{\prime}}, E_{\Sigma_{1}} \otimes E_{\Sigma_{1^{\prime}}}^{*} \otimes\left(\mathcal{N}_{\Sigma_{1} \cdot \Sigma_{1^{\prime}} \mid \Sigma_{1}} \otimes \mathcal{N}_{\Sigma_{1} \cdot \Sigma_{1^{\prime}} \mid \Sigma_{1^{\prime}}}\right)^{1 / 2}\right) \\
& -h^{0}\left(\Sigma_{1} \cdot \Sigma_{1^{\prime}}, E_{\Sigma_{1}}^{*} \otimes E_{\Sigma_{1^{\prime}}} \otimes\left(\mathcal{N}_{\Sigma_{1} \cdot \Sigma_{1^{\prime}} \mid \Sigma_{1}} \otimes \mathcal{N}_{\Sigma_{1} \cdot \Sigma_{1^{\prime}} \mid \Sigma_{1^{\prime}}}\right)^{1 / 2}\right) \\
= & h^{0}\left(\Sigma_{1} \cdot \Sigma_{1^{\prime}}, E_{\Sigma_{1}} \otimes E_{\Sigma_{1^{\prime}}}^{*} \otimes T^{*}\left(\Sigma_{1} \cdot \Sigma_{1^{\prime}}\right)^{1 / 2}\right)-h^{1}\left(\Sigma_{1} \cdot \Sigma_{1^{\prime}}, E_{\Sigma_{1}} \otimes E_{\Sigma_{1^{\prime}}}^{*} \otimes T^{*}\left(\Sigma_{1} \cdot \Sigma_{1^{\prime}}\right)^{1 / 2}\right) \\
= & \chi\left(\Sigma_{1} \cdot \Sigma_{1^{\prime}}, E_{\Sigma_{1}} \otimes E_{\Sigma_{1^{\prime}}}^{*} \otimes T^{*}\left(\Sigma_{1} \cdot \Sigma_{1^{\prime}}\right)^{1 / 2}\right) \\
= & \int_{\Sigma_{1} \cdot \Sigma_{1^{\prime}}} \operatorname{ch}\left(E_{\Sigma_{1}} \otimes E_{\Sigma_{1^{\prime}}}^{*}\right) e^{-\frac{1}{2} c_{1}\left(T\left(\Sigma_{1} \cdot \Sigma_{1^{\prime}}\right)\right)} \operatorname{Td}\left(T\left(\Sigma_{1} \cdot \Sigma_{1^{\prime}}\right)\right)=\int_{\Sigma_{1} \cdot \Sigma_{1^{\prime}}}\left(\frac{F_{\Sigma_{1}}}{2 \pi}-\frac{F_{\Sigma_{1^{\prime}}}}{2 \pi}\right)
\end{aligned}
$$

where the Calabi-Yau condition $T^{*}\left(\Sigma_{1} \cdot \Sigma_{1^{\prime}}\right) \simeq \mathcal{N}_{\Sigma_{1} \cdot \Sigma_{1^{\prime}} \mid \Sigma_{1^{\prime}}} \otimes \mathcal{N}_{\Sigma_{1_{1}} \cdot \Sigma_{1^{\prime}} \mid \Sigma_{1}}$ and the Serre duality are used in the second equality, and the last equality is the Hirzebruch-Riemann-Roch formula [50]. Here, $\chi, \mathrm{ch}$, and Td are the Euler characteristic, Chern character, and Todd class of complex vector bundles ${ }^{11}$. The number of chiral fermions is given by

$$
\begin{aligned}
N_{F} & \left.=\operatorname{index} \Sigma_{\Sigma_{1} \cdot \Sigma_{1^{\prime}}} \not D_{\left(E_{\Sigma_{1}} \otimes E_{\Sigma_{1^{\prime}}}^{*}\right.}\right) \\
& =\int_{\Sigma_{1} \cdot \Sigma_{1^{\prime}}} \operatorname{ch}\left(E_{\Sigma_{1}} \otimes E_{\Sigma_{1^{\prime}}}^{*}\right) \hat{A}\left(T\left(\Sigma_{1} \cdot \Sigma_{1^{\prime}}\right)\right) \\
& =\int_{\Sigma_{1} \cdot \Sigma_{1^{\prime}}} \operatorname{ch}\left(E_{\Sigma_{1}} \otimes E_{\Sigma_{1^{\prime}}}^{*}\right) e^{-\frac{1}{2} c_{1}\left(T\left(\Sigma_{1} \cdot \Sigma_{1^{\prime}}\right)\right)} \operatorname{Td}\left(T\left(\Sigma_{1} \cdot \Sigma_{1^{\prime}}\right)\right)=\int_{\Sigma_{1^{\prime}} \cdot \Sigma_{1^{\prime}}}\left(\frac{F_{\Sigma_{1}}}{2 \pi}-\frac{F_{\Sigma_{1^{\prime}}}}{2 \pi}\right),
\end{aligned}
$$

where the first line stands for the index of the Dirac operator on the curve $\Sigma_{1} \cdot \Sigma_{1^{\prime}}$, and $\hat{A}$ is the $\hat{A}$ class $^{12}$. Therefore, the number of massless chiral bosonic modes agrees with that of fermionic modes, as expected from $\mathrm{D}=4 \mathcal{N}=1 \mathrm{SUSY}$, and $N_{B}=N_{F}$ gives the number of families of chiral multiplets for each irreducible sub-bundle (sub-representation) of the Chan-Paton bundle $E_{\Sigma_{1}} \otimes E_{\Sigma_{1^{\prime}}}^{*}$. Note that all of $E_{\Sigma_{1}}$ and $E_{\Sigma_{1^{\prime}}}$ in (19) and (20) should be,

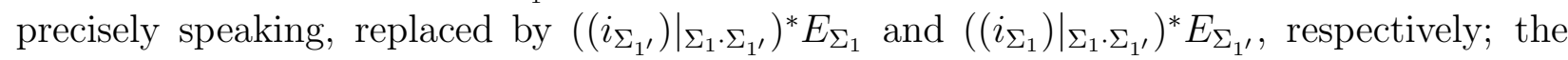
abuse of notations is just for visual clarity. The formulae (19) and (20) are nothing but a

\footnotetext{
${ }^{11}$ For their definitions, see [50].

${ }^{12}$ See [50] for definition.
} 
local version of the formula of the number of chiral multiplets in [51, where it is given by paring of the Ramond-Ramond charges of the D-branes [52, 53, 45, 59, 48, 60]. For the relation between the formula in [51] and (1920), see the appendix B.

The formula of the number of chiral multiplets (1920), or equivalently, (39) in the appendix $\mathrm{B}$, is applied to two stacks of ordinary D-branes. The multiplicity ${ }^{13}$ of chiral multiplets in the anti-symmetric representation that arise at the intersection of a stack of D7-branes and an O7-plane (and the mirror-image stack of D7-branes) [54, 55] is given in local form [56, 67] by (see also the appendix B)

$$
\frac{1}{2}\left[\int_{\Sigma_{1} \cdot \Sigma_{1^{\prime}}}\left(\frac{F_{\Sigma_{1}}}{2 \pi}-\frac{F_{\Sigma_{1^{\prime}}}}{2 \pi}\right)-\int_{\Sigma_{1} \cdot W} 2\left(\frac{F_{\Sigma_{1}}}{2 \pi}-\frac{B}{(2 \pi)^{2} \alpha^{\prime}}\right)\right]=\int_{\Sigma_{1} \cdot W} 2\left(\frac{F_{\Sigma_{1}}}{2 \pi}-\frac{B}{(2 \pi)^{2} \alpha^{\prime}}\right) .
$$

Here, $W$ is the locus of an O7-plane. This can be an odd integer such as 3 , since the $B$ field on a curve on an O7-plane is either integral or half integral [56, 68.

Let us turn our attention to the chiral multiplets in the $5^{*}$ representation. They are obtained just in the same way as the chiral multiplets in the $\mathbf{1 0}$ representation are obtained. To be more explicit, another D7-brane wrapped on a holomorphic 4-cycle $\Sigma_{2}$ is introduced, and $\Sigma_{2}$ is assumed to intersect with $\Sigma_{1}$ on a complex curve $\Sigma_{1} \cdot \Sigma_{2}$ (see Fig. 4). Massless particles in the $\left(\mathbf{5}, \mathbf{1}^{*}\right)\left(\right.$ or $\left.\left(\mathbf{5}^{*}, \mathbf{1}\right)\right)$ representation arise at the intersection from the open strings connecting the D7-branes on $\Sigma_{1}$ and $\Sigma_{2}$. After the Kaluza-Klein reduction on the complex curve, $\mathrm{D}=4$ massless matter contents can be chiral when the Chan-Paton bundles on $\Sigma_{1}$ and $\Sigma_{2}$ are suitably chosen. The number of massless chiral multiplets in the $5^{*}$ representation is given by (1920) with $\Sigma_{1^{\prime}}$ replaced by $\Sigma_{2}$.

The mechanism to obtain chiral matter discussed so far is essentially the one in [51, 55].

A U(1) symmetry arises from the D7-brane wrapped on $\Sigma_{2}$, which is denoted by $\mathrm{U}(1)_{0}$. Thus, the chiral multiplets obtained above is not in the $5^{*}$ representation, but in a bifundamental representation $\left(\mathbf{5}^{*}, \mathbf{1}\right)$ under $\mathrm{SU}(5)_{\mathrm{GUT}} \times \mathrm{U}(1)_{0}$. However, this is not a problem. The $\mathrm{U}(1)_{0}$ symmetry has mixed anomaly with $\mathrm{SU}(5)_{\mathrm{GUT}}$ because of the chiral multiplets. All the anomalous $\mathrm{U}(1)$ vector multiplets become massive through the Green-Schwarz interactions, and are irrelevant to the low-energy physics [57]. Likewise, the centre-of-mass $\mathrm{U}(1)$ symmetry arising from the first five D7-branes on $\Sigma_{1}$ is denoted by $U(1)_{5}$, and the $\mathrm{U}(1)$ symmetry from the sixth D7-brane on $\Sigma_{1}$ by $\mathrm{U}(1)_{6}$ (and the one from the seventh D7-brane on $\Sigma_{1}$ by $\mathrm{U}(1)_{7}$, if it exists). They also have mixed anomaly with $\mathrm{SU}(5)_{\text {GUT }}$. Some of linear combinations of these $\mathrm{U}(1)$ symmetries can be free from anomaly, and have corresponding

\footnotetext{
${ }^{13}$ New material added in version 2, March, 2006.
} 


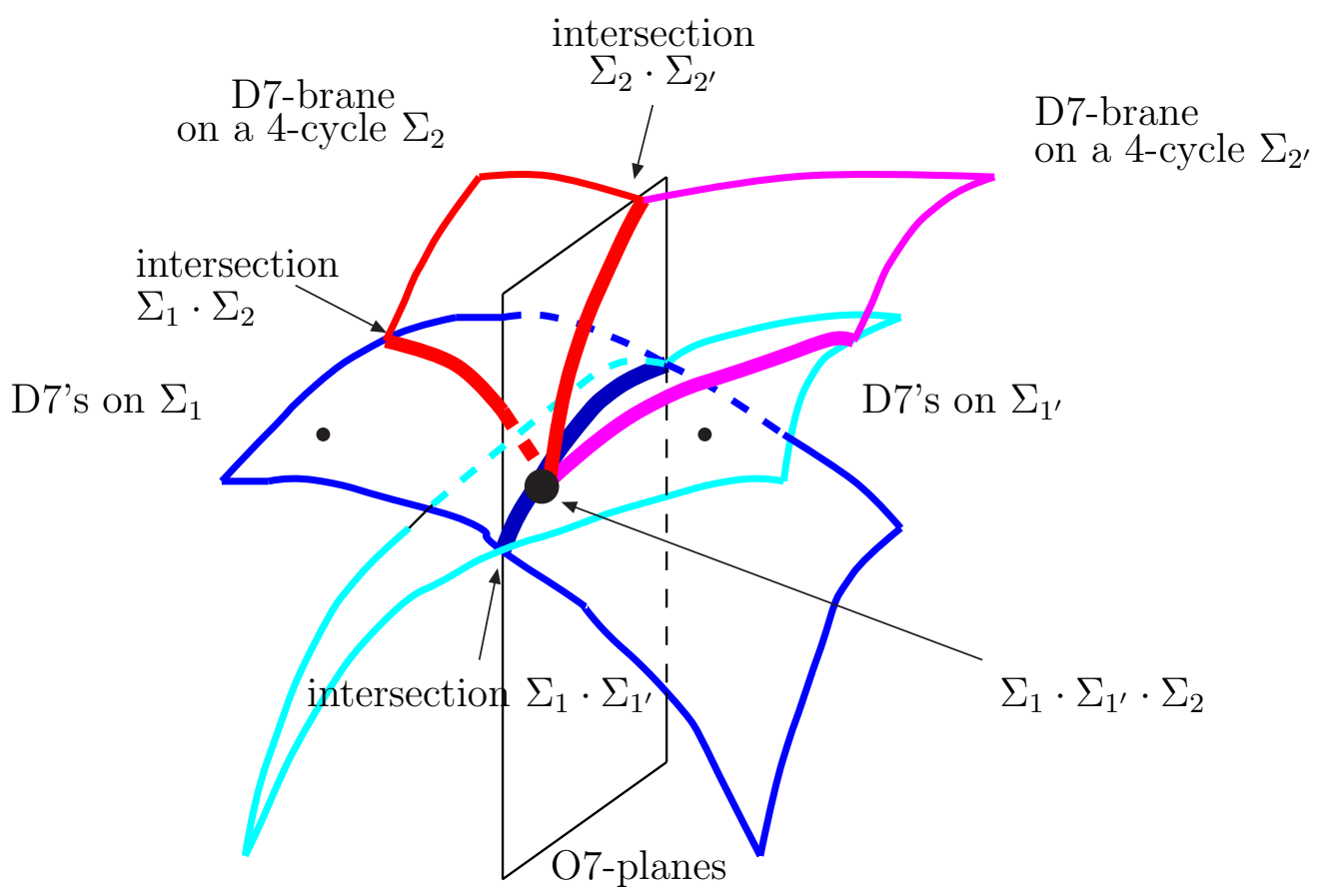

Figure 4: A schematic picture of the D-brane configuration for quarks and leptons. The $\mathrm{SU}(5)_{\text {GUT }}$ gauge field propagates on the D7-branes on $\Sigma_{1}$ and $\Sigma_{1^{\prime}}$. The chiral multiplets in the 10 representation are localized on $\Sigma_{1} \cdot \Sigma_{1^{\prime}}$, and those in the $5^{*}$ representation (and right-handed neutrinos) on $\Sigma_{1} \cdot \Sigma_{2}$. The GUT-breaking sector is denoted by a small dot on $\Sigma_{1}$ (and its orientifold image on $\Sigma_{1^{\prime}}$ ).

massless gauge fields. Let us consider the following linear combination, which is free from the mixed anomaly with $\mathrm{SU}(5)_{\mathrm{GUT}}$ :

$$
1 \times U(1)_{5}+(-5) \times U(1)_{0}+5 \times U(1)_{6} \quad\left(+5 \times U(1)_{7}+\cdots\right) .
$$

The 10 representation is charged by $+2,5^{*}$ by $-6, \bar{H}_{i}^{6}\left(\mathbf{5}^{*}\right)$ by +4 , and $H^{i}{ }_{7}$ or $6(5)$ by -4 under this symmetry, and hence we identify this symmetry with the $\mathrm{U}(1)_{B-L}$ symmetry. The triangle anomaly of itself vanishes when there are three chiral multiplets whose $\mathrm{U}(1)_{B-L}$ charge is -10 . These multiplets are identified with right-handed neutrinos. Since the open strings connecting the D7-brane on $\Sigma_{2}$ and the sixth (or seventh) D7-brane on $\Sigma_{1}$ carries $\mathrm{U}(1)_{B-L}$ charge by \pm 10 , we expect the right-handed neutrinos arise from those strings. Therefore, it follows that the chiral multiplets in the $\mathbf{5}^{*}$ representation and the right-handed neutrinos are localized at the intersection $\Sigma_{1} \cdot \Sigma_{2}$, whereas those in the $\mathbf{1 0}$ representation are at the intersection $\Sigma_{1} \cdot \Sigma_{1^{\prime}}$ (Fig. 41). 


\subsubsection{Ramond-Ramond Charge Cancellation and Origin of Family Structure}

We have not discussed the cancellation of the Ramond-Ramond charges of D-branes. This subsubsection is devoted in describing, in a qualitative manner, how the charges can be cancelled. We do not try to obtain an explicit D-brane configuration and background geometry in this article ${ }^{14}$. Although some of phenomenological aspects depend on the explicit solutions, there are also some generic features that do not depend on such details. One will see at the end of this subsubsection that the qualitative understanding of the Ramond-Ramond charge cancellation suggests us a possible geometric origin of family structure of quarks and leptons.

The Bianchi identities of the Ramond-Ramond potentials are given by $\left[58,\left[53\right.\right.$, 59, 48] ${ }^{15}$

$$
d G=\sum_{k} v_{X}\left(\Sigma_{k}, E_{\Sigma_{k}}\right),
$$

where $G$ is the sum of field strengths of the various Ramond-Ramond potentials and $v_{X}\left(\Sigma_{k}, E_{\Sigma_{k}}\right)$ is the Ramond-Ramond charge of a D-brane wrapped on a cycle $\Sigma_{k}$. $E_{\Sigma_{k}}$ stands for the Chan-Paton bundle on it. The explicit expression for the Ramond-Ramond charge [52, 53, 45, 59, 48, 60] is given in (36137) in the appendix B for convenience. The both sides of (231) are in even-dimensional cohomology of the Calabi-Yau 3-fold. Now, the both sides are integrated on an even-dimensional cycle, and then, the Ramond-Ramond charge cancellation condition follows:

$$
\int_{\text {compact cycle of } X} \sum_{k} v_{X}\left(\Sigma_{k}, E_{\Sigma_{k}}\right)=0 .
$$

We are not interested in the condition (24) for the total Calabi-Yau 3-fold (6-cycle), since we are concerned only about the local model. Conditions only for compact 2-cycles and 4-cycles relevant to our construction are considered in the following.

The intersection of two holomorphic 4-cycles $\Sigma$ and $\Sigma^{\prime}$ is a compact 2-cycle $\Sigma \cdot \Sigma^{\prime}$, and hence we have a charge cancellation condition associated with this cycle. This type of condition is applied for $\Sigma_{1} \cdot \Sigma_{1^{\prime}}, \Sigma_{1} \cdot \Sigma_{2}$, etc.. A D7-brane wrapped on $\Sigma^{\prime}$ contributes to the condition for $\Sigma \cdot \Sigma^{\prime}$ by

$$
\int_{\Sigma \cdot \Sigma^{\prime}}\left(i_{\Sigma^{\prime}}\right)_{*}(1)=\#\left(\Sigma \cdot \Sigma^{\prime} \cdot \Sigma^{\prime}\right),
$$

\footnotetext{
${ }^{14}$ Thus, it is not guaranteed that there is a consistent solution that realizes the idea described in this article. We just assume in this article that there is.

${ }^{15}$ Contributions from the background fluxes are not taken into account here.
} 
i.e., by the intersection number of $\Sigma, \Sigma^{\prime}$, and again, $\Sigma^{\prime} . N$ D7-branes wrapped on $\Sigma$ contribute to the charge cancellation condition by $N \times \#\left(\Sigma \cdot \Sigma^{\prime} \cdot \Sigma\right)$. These contributions are, in general, non-zero, and have to be cancelled by other contributions from D7-branes wrapped on other 4-cycles. A D7-brane wrapped on a 4-cycle $\Sigma^{\prime \prime}$ contribute by the intersection number $\#\left(\Sigma \cdot \Sigma^{\prime} \cdot \Sigma^{\prime \prime}\right)$.

A compact 4-cycle $\Sigma$ also has a condition, which is obtained by integrating the Bianchi identity (23) over it. This type of condition is applied for $\Sigma_{1}$ and $\Sigma_{2}$. D7-branes wrapped on $\Sigma$ itself contribute by ${ }^{16,17}$

$$
\begin{aligned}
\int_{\Sigma}\left(i_{\Sigma}\right)_{*}\left(\operatorname{ch}\left(E_{\Sigma} \otimes \mathcal{L}_{B}^{-1}\right)\right)_{2-\text { form }}= & \int_{\Sigma} c_{1}\left(\mathcal{N}_{\Sigma \mid X}\right) \wedge\left(\operatorname{ch}\left(E_{\Sigma} \otimes \mathcal{L}_{B}^{-1}\right)\right)_{2-\text { form }} \\
& =\int_{\Sigma \cdot \Sigma}\left(\frac{F_{\Sigma}}{2 \pi}-\frac{B}{(2 \pi)^{2} \alpha^{\prime}}\right) .
\end{aligned}
$$

Other D7-branes (wrapped on a 4-cycle $\Sigma^{\prime \prime \prime}$ ) contribute by

$$
\begin{aligned}
\int_{\Sigma}\left(i_{\Sigma^{\prime \prime \prime}}\right)_{*}\left(\operatorname{ch}\left(E_{\Sigma^{\prime \prime \prime}} \otimes \mathcal{L}_{B}^{-1}\right)\right)_{2-\text { form }}= & \int_{\Sigma \cdot \Sigma^{\prime \prime \prime}}\left(\left.\left(i_{\Sigma}\right)\right|_{\Sigma \cdot \Sigma^{\prime \prime \prime}}\right)^{*}\left(\operatorname{ch}\left(E_{\Sigma^{\prime \prime \prime}} \otimes \mathcal{L}_{B}^{-1}\right)\right)_{2-\text { form }} \\
& =\int_{\Sigma \cdot \Sigma^{\prime \prime \prime}}\left(\frac{F_{\Sigma^{\prime \prime \prime}}}{2 \pi}-\frac{B}{(2 \pi)^{2} \alpha^{\prime}}\right) .
\end{aligned}
$$

Here, $\mathcal{L}_{B}$ is a "line bundle" whose first Chern class is $B /\left((2 \pi)^{2} \alpha^{\prime}\right)$. D5-branes, including the fractional D3-branes, also contribute to the condition. These contributions have to cancel one another for each 4-cycle. Notice that the effects of the orientifold projection has not been considered seriously, and thus the above argument is only qualitative.

The Chern-Simons interaction on D-branes guarantees that the theory is locally anomaly free at any points of the $\mathrm{D}=10$ Type IIB string theory. Localization of fermions and that of Ramond-Ramond charge are related through the Chern-Simons interaction, and the charge gives rise to proper inflow of anomaly for the localized fermion [53, 59, 48, 60]. Therefore, D $=4$ theories obtained after compactification are free from anomalies, as long as the total sum of the anomaly inflow vanishes, or in other words, as long as the sum of Ramond-Ramond charges vanishes on every compact cycle. The Ramond-Ramond charge cancellation on 4cycles is responsible for the triangle anomalies on the D7 world volumes, and the cancellation on 2-cycles both for triangle anomalies on D5 and box anomalies on D7 world volumes.

Incidentally, there have been proposed phenomenological models of the family structure of quarks and leptons that use anomaly inflow and cancellation in the internal space 61, 62.

\footnotetext{
${ }^{16}$ The self-intersection formula for hypersurface $\left(i_{\Sigma}\right)^{*}\left(i_{\Sigma}\right)_{*}(1)=c_{1}\left(\mathcal{N}_{\Sigma \mid X}\right)$ is used.

${ }^{17}$ The expression (37) is used for the Ramond-Ramond charge of D-branes. See also footnote 17.
} 

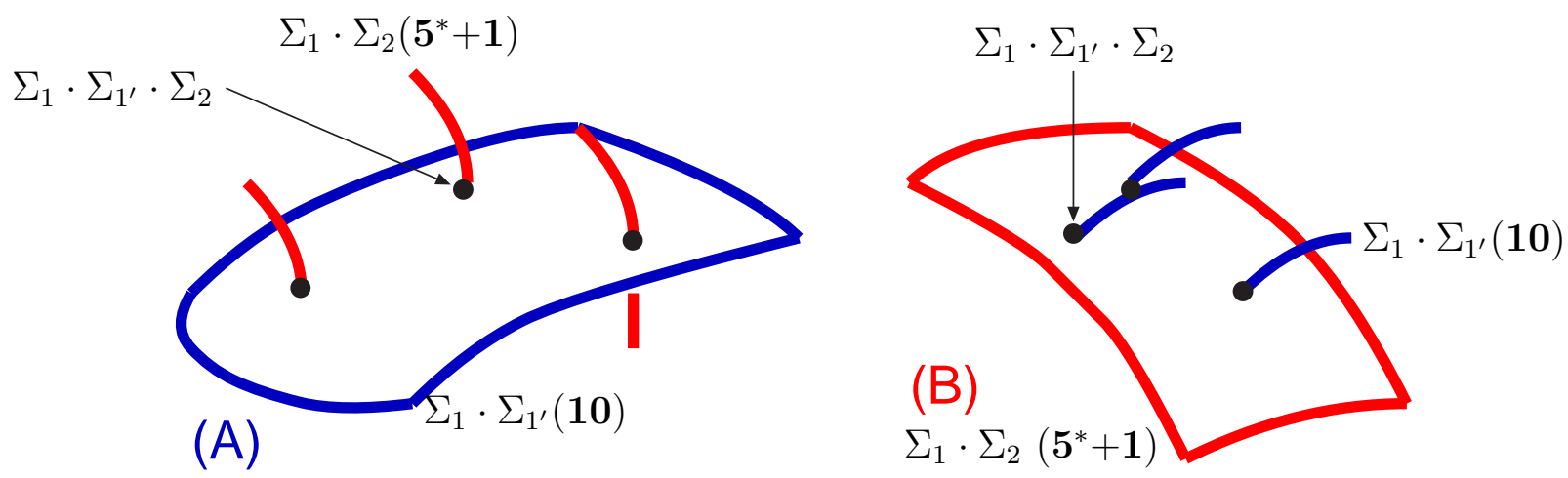

Figure 5: Phenomenological models of family structure in 61 can be considered as effective theories obtained by projecting onto the complex curve $\Sigma_{1} \cdot \Sigma_{1^{\prime}}(\mathrm{A})$. Multiplets in the $5^{*}$ representation and right-handed neutrinos are localized in the internal space, and the large mixing between them may be understood in terms of geometry. Another model in [62] can be considered as an effective theory obtained by projecting onto the complex curve $\Sigma_{1} \cdot \Sigma_{2}$ (B). Multiplets in the $\mathbf{1 0}$ representation are localized in the internal space, and the large hierarchy between them may be explained geometrically.

It is one of the biggest mysteries in the context of unified theories why and how the mixing angles of the $\mathrm{SU}(2)_{L}$ interaction are small in the quark sector while large in the lepton sector [63, 64, 65, 66]. Models in 61, 62] are phenomenological approaches to this mystery. Chiral multiplets in the $\mathrm{SU}(5)_{\mathrm{GUT}^{-}} \mathbf{1 0}$ representation and those in the $\mathrm{SU}(5)_{\mathrm{GUT}^{-}} \mathbf{5}^{*}$ representation are assumed to have totally different wavefunctions (i.e., localization properties). Proposed there are ideas of geometric realization of the origin of non-parallel family structure [22, 23. desired phenomenologically.

It is a remarkable coincidence that the D-brane configuration obtained in this article happens to support the phenomenologically motivated models of the family structure [61, 62; the models were proposed totally independent from the D-brane realization of unified theories considered in this article. The multiplets in the $\mathbf{1 0}$ representation are localized on the intersection $\Sigma_{1} \cdot \Sigma_{1^{\prime}}$, while those in $5^{*}$ and the right-handed neutrinos are on $\Sigma_{1} \cdot \Sigma_{2}$. Let us suppose that the hypersurfaces $\Sigma_{1}, \Sigma_{1^{\prime}}$ and $\Sigma_{2}$ have non-vanishing intersection number, i.e., $\#\left(\Sigma_{1} \cdot \Sigma_{1^{\prime}} \cdot \Sigma_{2}\right) \neq 0$. Then, models in [61] can be considered as the effective theories obtained by projecting the model in this article onto the complex curve $\Sigma_{1} \cdot \Sigma_{1^{\prime}}$ (Fig. 5 (A)). There, the anomaly due to the chiral zero modes in the $\mathbf{1 0}$ representation on the complex curve $\Sigma_{1} \cdot \Sigma_{1^{\prime}}$ flows into some points $\Sigma_{1} \cdot \Sigma_{1^{\prime}} \cdot \Sigma_{2}$, where the anomaly is cancelled by chiral 
zero modes in the $5^{*}$ representation and the right-handed neutrinos. The models in [62] can be considered as the effective theory obtained by projecting the model in this article onto the complex curve $\Sigma_{1} \cdot \Sigma_{2}$ (Fig. [5 (B)). There, the anomaly due to the chiral zero modes in $5^{*}$ and the right-handed neutrinos flows into some points $\Sigma_{1} \cdot \Sigma_{1^{\prime}} \cdot \Sigma_{2}$, where the anomaly is cancelled by chiral zero modes in the $\mathbf{1 0}$ representation. Therefore, the framework in this subsection, in which 10's and $5^{*}$ 's are obtained at (different) intersections of D7-branes, not only exhibits the origin of the non-parallel family structure, but also (hopefully) makes it possible to obtain better understanding of the origin of the family structure and Yukawa coupling of all the quarks and leptons.

\section{Summary and Open Questions}

The absence of the large mass for the two Higgs doublets and the suppressed dimensions-five proton decay give us important clues to how the unified theory would be. The productgroup unification models, based on $\mathrm{SU}(5)_{\mathrm{GUT}} \times \mathrm{U}(N)_{\mathrm{H}}$ gauge group $(N=2,3)$, are one of a few classes of theories in which the two clues above are understood in terms of a single symmetry. A preceding work [17] suggests that the brane-world picture may exist behind the models. Based on this motivation, we have illustrated an idea of how to embed the model into the Type IIB string theory.

The Type IIB string theory is compactified on an orientifolded Calabi-Yau 3-fold, and space-filling D-branes are wrapped on holomorphic cycles of the Calabi-Yau 3-fold, so that $\mathrm{D}=4 \mathcal{N}=1$ SUSY is preserved. Various particles of the model, including quarks and leptons, and particles in the GUT-symmetry-breaking sector, are obtained as the massless modes contained in open strings connecting those D-branes. We do not restrict ourselves to toroidal orbifolds as the candidates of the Calabi-Yau 3-fold. We allow ourselves to choose a generic curved Calabi-Yau manifold, so that we can hope to find a suitable manifold that accommodates all the particles we need. Although one can no longer hope to calculate everything without a CFT formulation, it is certainly not our primary interest. Instead, local geometry and local D-brane configuration are determined so that phenomenologically desired particle contents are obtained. Once the local configuration is fixed, then it may be possible to derive some new phenomenological implications, if not predictions; local information is sufficient for limited purposes. This is what we hope to do.

The GUT-breaking sector is realized by a D3-D7 system put on the local geometry $\mathbf{C}^{2} / \mathbf{Z}_{M} \times \mathbf{C}$. The $\mathrm{U}(N)_{\mathrm{H}}$ gauge group is realized by fractional D3-branes. The $\mathrm{SU}(5)_{\text {GUT }}$ 
gauge group is realized by D7-branes wrapped on a 4-cycle $\Sigma_{1}$. Phenomenology requires that $\operatorname{vol}\left(\Sigma_{1}\right) /\left(2 \pi \sqrt{\alpha^{\prime}}\right)^{4} \sim 10-100$ and $g_{s} \sim(1 / 2-2)$. The total volume is determined by $\operatorname{vol}\left(C Y_{3}\right) / \operatorname{vol}\left(\Sigma_{1}\right) /\left(2 \pi \sqrt{\alpha^{\prime}}\right)^{2} \sim 100$, when the string scale $1 / \sqrt{\alpha^{\prime}}$ is of the order of $10^{17} \mathrm{GeV}$. Although the origin of the GUT scale is not clarified in this article, it may be related to the Kaluza-Klein scale.

The $\mathrm{SU}(5)_{\mathrm{GUT}} \times \mathrm{U}(3)_{\mathrm{H}}$ model [13] contains Higgs multiplets, which are fundamental and anti-fundamental representations of $\mathrm{SU}(5)_{\text {GUT }}$. This model realizes the doublet-triplet splitting through the missing partner mechanism, and hence, there should be sizable coupling between the Higgs multiplets and the particles in the GUT-symmetry-breaking sector. Therefore, it is both economical and phenomenologically desirable, if the Higgs particles are obtained from open strings connecting the D7-branes wrapped on $\Sigma_{1}$. If a geometry around the 4-cycle $\Sigma_{1}$ satisfies a particular condition, then the two Higgs multiplets are obtained from six D7-branes. If the condition is not satisfied, one can obtain them from seven D7-branes. The gauge singlet of the NMSSM and its tri-linear interaction with the two Higgs doublets can also be realized easily as a special case.

The chiral quarks and leptons are obtained at intersections of two stacks of D7-branes. Chiral multiplets in the $\mathbf{1 0}$ representation are obtained at the intersection of five D7-branes and their orientifold mirror images. Those in the $5^{*}$ representation and the right-handed neutrinos are obtained at an intersection of the five D7-branes and another D7-brane. D $=4$ chiral theories are obtained if the Chan-Paton bundles on the D7-branes are suitably chosen. The number of family is given by the pairing of the Ramond-Ramond charges of the D-branes (and orientifold planes).

The multiplets in the $\mathbf{1 0}$ representation are localized in one D7-D7 intersection and those in the $5^{*}$ representation and the right-handed neutrinos are at another. One of the important consequences is that this configuration leads to a geometric realization of the non-parallel family structure, which was proposed to explain the large mixing angles in the neutrino oscillation. The other important consequence is that the rate of the proton decay through dimension-six operators is enhanced, and the branching ratios are modified, as in [38.

In this article, we have discussed properties that local configuration has to satisfy, and have illustrated an idea of how to construct a model that satisfies those properties. But, this article does not present an explicit model where the Ramond-Ramond charges are cancelled at all the cycles relevant to the model. That is, the existence of the consistent solution is not guaranteed. If one further pursues to find an explicit solution, one might be able to predict extra particles (e.g., such as those in [26]) that can be observable in future detectors. 
One might also be able to determine whether the singlet of the NMSSM really exists in the spectrum or not. It may also be that the idea of lifting the $\mathrm{D}=4$ models to the Type IIB string theory is excluded because of the absence of a consistent solution.

The origin of Yukawa coupling and other interactions in the superpotential is also poorly understood. Some of them are obtained through perturbative interactions of the Type IIB theory, but not all of them. Although the discrete $R$ symmetry plays quite an important role in the $\mathrm{D}=4$ models, the origin of this symmetry is not identified, either. We leave these issues to further investigation.

\section{Acknowledgements}

The authors are grateful to the Theory Division of CERN for the hospitality, where earlier part of this work was done. T.W. thanks the Japan Society for the Promotion of Science and the Miller Institute for the Basic Research of Science. This work is partially supported by the Director, Office of Science, Office of High Energy and Nuclear Physics, of the U.S. Department of Energy under Contract DE-AC03-76SF00098 (T.W.), and Grant-in-Aid Scientific Research (s) 14102004 (T.Y.).

\section{A Toy Model of Chiral Anti-Symmetric-Tensor Repre- sentation at the Intersection of D7-branes}

This appendix provides details of the toy model sketched in subsubsection 3.3.1. One can see from the model how chiral matter in anti-symmetric representation is obtained at the intersection of two stacks of D7-branes.

We first consider a background geometry $\mathbf{R}^{3,1} \times \mathbf{T}^{2} \times \mathbf{C}^{2}$. Coordinates $x^{0,1,2,3}$, $z^{1}$, and $\left(z^{2} \equiv\left(x^{6}+i x^{7}\right), z^{3} \equiv\left(x^{8}+i x^{9}\right)\right)$ are used for $\mathbf{R}^{3,1}, \mathbf{T}^{2}$ and $\mathbf{C}^{2}$, respectively. The "internal" space is $\mathbf{T}^{2} \times \mathbf{C}^{2}$ and is non-compact. The non-compact geometry is regarded as a local geometry around the intersection of D7-branes we are interested in. Two stacks of $\mathbf{R}^{3,1}$ filling D7-branes are introduced. One of them, which we refer to as D7 , is stretched in $z^{1} \wedge \bar{z}^{1} \wedge\left(\cos \theta z^{2}-\sin \theta z^{3}\right) \wedge\left(\cos \theta \bar{z}^{2}-\sin \theta \bar{z}^{3}\right)$, and the other, which we refer to as D7+, in $z^{1} \wedge \bar{z}^{1} \wedge\left(\cos \theta z^{2}+\sin \theta z^{3}\right) \wedge\left(\cos \theta \bar{z}^{2}+\sin \theta \bar{z}^{3}\right)$. They intersect at a complex curve defined by $\left(z^{2}, z^{3}\right)=(0,0)$.

The mode expansion of the open strings connecting $\mathrm{D} 7_{ \pm}$to $\mathrm{D} 7_{ \pm}$itself is the same as that of the flat D7-branes. The fluctuation in $x^{0,1,2,3}$ and $\mathbf{T}^{2}$-directions have the same mode 
expansion also for the open strings connecting $\mathrm{D} 7_{\mp}$ to $\mathrm{D} 7_{ \pm}$. However, the fluctuation in $z_{2}$ and $z_{3}$ directions have different mode expansion. The boundary condition is given by

$$
\begin{array}{ll}
\partial_{\sigma} \operatorname{Re}\left(e^{i \theta}\left(X^{6}+i X^{8}\right)\right)=0, & \partial_{\sigma} \operatorname{Re}\left(e^{i \theta}\left(X^{7}+i X^{9}\right)\right)=0, \\
\partial_{\tau} \operatorname{Im}\left(e^{i \theta}\left(X^{6}+i X^{8}\right)\right)=0, & \partial_{\tau} \operatorname{Im}\left(e^{i \theta}\left(X^{7}+i X^{9}\right)\right)=0,
\end{array}
$$

at $\sigma=0$ for the open strings starting from $\mathrm{D} 7_{-}$and ending on $\mathrm{D} 7_{+} \cdot e^{i \theta}$ is replaced by $e^{-i \theta}$ in the boundary conditions at $\sigma=\pi$. The following mode expansion satisfies the above boundary conditions:

$$
\begin{aligned}
X^{6}+i X^{8} & =i\left(\frac{\alpha^{\prime}}{2}\right)^{\frac{1}{2}} \sum_{m \in \mathbb{Z}}\left(\frac{\alpha_{m+v}^{(6,8)}}{m+v} e^{-i(m+v)(\tau-\sigma)} e^{-i \theta}+\frac{\beta_{m-v}^{(6,8)}}{m-v} e^{-i(m-v)(\tau+\sigma)} e^{-i \theta}\right), \\
X^{6}-i X^{8} & =i\left(\frac{\alpha^{\prime}}{2}\right)^{\frac{1}{2}} \sum_{m \in \mathbb{Z}}\left(\frac{\beta_{m-v}^{(6,8)}}{m-v} e^{-i(m-v)(\tau-\sigma)} e^{i \theta}+\frac{\alpha_{m+v}^{(6,8)}}{m+v} e^{-i(m+v)(\tau+\sigma)} e^{i \theta}\right)
\end{aligned}
$$

where $v \equiv 2 \theta / \pi$, and oscillators $\alpha_{m+v}^{(6,8)}$ and $\beta_{m-v}^{(6,8)}$ satisfy $\left(\alpha_{m+v}^{(6,8)}\right)^{\dagger}=\beta_{-m-v}^{(6,8)}$. The mode expansion of $X^{7} \pm i X^{9}$ is exactly the same except that the oscillators $\alpha_{m+v}^{(6,8)}$ and $\beta_{m-v}^{(6,8)}$ are replaced by $\alpha_{m+v}^{(7,9)}$ and $\beta_{m-v}^{(7,9)}$. The mode expansion of the world-sheet fermions is determined from that of the world-sheet bosons given above:

$$
\begin{aligned}
\psi^{(6,8)} & =\sum_{r \in \mathbb{Z}+\frac{1}{2} \text { or } \mathbb{Z}} \psi_{r+v}^{(6,8)} e^{-i(r+v)(\tau-\sigma)} e^{-i \theta}, & \tilde{\psi}^{(6,8)} & =\sum_{r \in \mathbb{Z}+\frac{1}{2} \text { or } \mathbb{Z}} \bar{\psi}_{r-v}^{(6,8)} e^{-i(r-v)(\tau+\sigma)} e^{-i \theta}, \\
\bar{\psi}^{(6,8)} & =\sum_{r \in \mathbb{Z}+\frac{1}{2} \text { or } \mathbb{Z}} \bar{\psi}_{r-v}^{(6,8)} e^{-i(r-v)(\tau-\sigma)} e^{i \theta}, & \tilde{\bar{\psi}}^{(6,8)} & =\sum_{r \in \mathbb{Z}+\frac{1}{2} \text { or } \mathbb{Z}} \psi_{r+v}^{(6,8)} e^{-i(r+v)(\tau+\sigma)} e^{i \theta} .
\end{aligned}
$$

Here, $r \in \mathbb{Z}+1 / 2$ for the NS sector and $\in \mathbb{Z}$ for the $\mathrm{R}$ sector.

The massless spectrum of the $\mathrm{D} 7_{ \pm}-\mathrm{D} 7_{ \pm}$open string is quite simple - that of the YangMills theory on a $7+1$-dimensional spacetime with 16 SUSY charges. The massless modes of the $\mathrm{D} 7_{\mp}-\mathrm{D} 7_{ \pm}$open string sector are localized at the intersection of $\mathrm{D} 7_{-}$and $\mathrm{D} 7_{+}$, unless $\theta$ is an integral multiple of $\pi / 2$. A hypermultiplet in the bi-fundamental representation is obtained there; four scalar bosons of the hypermultiplet are $\psi_{-\frac{1}{2}+v}^{(6,8)}\left|0 ; 7_{-} 7_{+} ; N S\right\rangle, \psi_{-\frac{1}{2}+v}^{(7,9)}\left|0 ; 7_{-} 7_{+} ; N S\right\rangle$, $\bar{\psi}_{-\frac{1}{2}+v}^{(6,8)}\left|0 ; 7_{+} 7_{-} ; N S\right\rangle$, and $\bar{\psi}_{-\frac{1}{2}+v}^{(7,9)}\left|0 ; 7_{+} 7_{-} ; N S\right\rangle$, while fermions are obtained from the Clifford algebra of $\psi_{0}^{(2,3)}, \psi_{0}^{(4,5)}, \bar{\psi}_{0}^{(2,3)}$ and $\bar{\psi}_{0}^{(4,5)}$ in the $\mathrm{R}$ sector. Here, we implicitly assume $0<\theta<$ $\pi / 4$ (i.e., $0<v<1 / 2$ ) just to avoid technical details.

Now let us impose an orientifold projection associated with $\Omega R_{z^{3}}(-1)^{F_{L}} \cdot R_{z^{3}}$ reflects the third complex plane, i.e., $R_{z^{3}}: z^{3} \mapsto-z^{3}$. Thus, we have an O7-plane at $z_{3}=0$, and $\mathrm{D} 7_{+}$ 
is the orientifold mirror image of the D7_ and vice versa. Therefore, the Yang-Mills fields on $\mathrm{D} 7_{-}$are identified with those on $\mathrm{D} 7_{+}$. On the other hand, the $\mathrm{D} 7_{\mp}-\mathrm{D} 7_{ \pm}$open strings are mapped to themselves, not to each other. Thus, the orientifold projection conditions are imposed:

$$
\begin{aligned}
\psi_{-\frac{1}{2}+v}^{(6,8) /(7,9)}\left|0 ; i_{-} j_{+} ; N S\right\rangle \sim & \left(\Omega R_{z^{3}}(-1)^{F_{L}}\right) \psi_{-\frac{1}{2}+v}^{(6,8) /(7,9)}\left|0 ; i_{-} j_{+} ; N S\right\rangle \\
= & -\psi_{-\frac{1}{2}+v}^{(6,8) /(7,9)}\left|0 ; j_{-} i_{+} ; N S\right\rangle
\end{aligned}
$$

for $0<\theta<\pi / 4$. As a result, we have a hypermultiplet in the second-rank anti-symmetrictensor representation.

An orbifold projection associated with a transformation

$$
z^{1} \mapsto e^{2 i \alpha} z^{1}, \quad z^{2} \mapsto e^{-i \alpha} z^{2}, \quad z^{3} \mapsto e^{-i \alpha} z^{3}
$$

is now imposed, so that chiral matter content is obtained in the four-dimensional effective field theory. $\alpha$ is an integral multiple of $2 \pi / N$, when the orbifold group is $\mathbf{Z}_{N}$. The oscillators $\psi_{-\frac{1}{2}+v}^{(6,8)} \pm i \psi_{-\frac{1}{2}+v}^{(7,9)}$, and $\bar{\psi}_{-\frac{1}{2}+v}^{(6,8)} \pm i \bar{\psi}_{-\frac{1}{2}+v}^{(7,9)}$ are multiplied by a phase $e^{\mp i \alpha}$ under the transformation. Suppose that the Chan-Paton matrix associated with the orbifold projection multiplies a phase $e^{i \beta}$ to $\mathrm{D} 7_{-}-\mathrm{D} 7_{+}$states and a phase $e^{-i \beta}$ to $\mathrm{D} 7_{+}-\mathrm{D} 7_{-}$states, then, two states $\left(\psi_{-\frac{1}{2}+v}^{(6,8)}+\right.$ $\left.i \psi_{-\frac{1}{2}+v}^{(7,9)}\right)\left|0 ; 7_{-} 7_{+} ; N S\right\rangle$ and $\left(\bar{\psi}_{-\frac{1}{2}+v}^{(6,8)}-i \bar{\psi}_{-\frac{1}{2}+v}^{(7,9)}\right)\left|0 ; 7_{+} 7_{-} ; N S\right\rangle$ satisfy the projection condition if $\alpha \equiv \beta$. Likewise, two states in the $\mathrm{R}$-sector $\mathrm{D} 7_{-}-\mathrm{D} 7_{+}$open string are rotated by $e^{ \pm i \alpha}$, because of the phase rotation of the oscillators $\psi_{0}^{(4,5)}$ and $\bar{\psi}_{0}^{(4,5)}$, and so are the two states in the R-sector $\mathrm{D} 7_{+}-\mathrm{D} 7_{-}$open string. Thus, one state from D7_-D7+ string and one state from $\mathrm{D} 7_{+}-\mathrm{D} 7_{-}$survive the orbifold projection condition when $\alpha \equiv \beta$. These two states from the NS sector and two states from the $\mathrm{R}$ sector form a chiral multiplet of the $\mathcal{N}=1$ SUSY of four-dimensional spacetime. This multiplet is a half of the hypermultiplet in the anti-symmetric representation, and the other half (i.e., the chiral multiplet in the conjugate representation) is projected out.

\section{B Global and Local Formulae of the Number of Chiral Families}

Quarks and leptons arise at the intersection of the two stacks of the D7-branes. The number of families in $\mathrm{D}=4$ effective theory is given by formula (1920), which only uses quantities defined locally around the intersection. Incidentally, the number of chiral multiplets in D 
$=4$ effective theory is obtained also through a formula in [51, which is given in terms of vector bundles on the whole Calabi-Yau 3-fold. The purpose of this appendix is to show the equivalence between them explicitly.

The Ramond-Ramond charges of D-branes are classified as elements of even-dimensional cohomology groups of the Calabi-Yau 3-fold $X$ [52, 53, 45, 59, 48, 60]:

$$
v_{X}\left(\Sigma, E_{\Sigma}\right)=\operatorname{ch}\left(\left(i_{\Sigma}\right) ! E_{\Sigma} \otimes \mathcal{L}_{B}^{-1}\right) \sqrt{\hat{A}(T X)} \in H^{\text {even }}(X),
$$

for a D-brane wrapped on a cycle $i_{\Sigma}: \Sigma \hookrightarrow X$ with Chan-Paton bundle $E_{\Sigma}$ on it, or equivalently,

$$
v_{X}\left(\Sigma, E_{\Sigma}\right)=\left(i_{\Sigma}\right)_{*}\left(\operatorname{ch}\left(E_{\Sigma} \otimes \mathcal{L}_{B}^{-1}\right) \sqrt{\frac{\hat{A}(T \Sigma)}{\hat{A}\left(\mathcal{N}_{\Sigma \mid X}\right)}}\right) \in H^{\text {even }}(X) .
$$

Here, $\left(i_{\Sigma}\right)_{*}$ and $\left(i_{\Sigma}\right)$ ! are the Thom isomorphism for cohomology and its analogue for vector bundles, respectively, associated with the embedding $i_{\Sigma}$. The pairing of the Ramond-Ramond charges is defined by

$$
I_{X}\left(\left(\Sigma_{1}, E_{\Sigma_{1}}\right),\left(\Sigma_{2}, E_{\Sigma_{2}}\right)\right)=\int_{X} v_{X}\left(\Sigma_{1}, E_{\Sigma_{1}}\right)^{\vee} \wedge v_{X}\left(\Sigma_{2}, E_{\Sigma_{2}}\right),
$$

where $v_{X}\left(\Sigma_{1}, E_{\Sigma_{1}}\right)^{\vee}$ is obtained from $v_{X}\left(\Sigma_{1}, E_{\Sigma_{1}}\right)$ by multiplying $(-1)^{q}$ to $2 q$-dimensional cohomology, or equivalently,

$$
\begin{aligned}
I_{X}\left(\left(\Sigma_{1}, E_{\Sigma_{1}}\right),\left(\Sigma_{2}, E_{\Sigma_{2}}\right)\right) & =\int_{X} \operatorname{ch}\left[\left(\left(i_{\Sigma_{1}}\right) ! E_{\Sigma_{1}} \otimes \mathcal{L}_{B}^{-1}\right)^{*} \otimes\left(\left(i_{\Sigma_{2}}\right) ! E_{\Sigma_{2}} \otimes \mathcal{L}_{B}^{-1}\right)\right] \hat{A}(T X) \\
& =\operatorname{index}_{X} \not D\left(\left(i_{\Sigma_{1}}\right) E_{\Sigma_{1}} \otimes \mathcal{L}_{B}^{-1}\right)^{*} \otimes\left(\left(i_{\Sigma_{2}}\right) ! E_{\Sigma_{2}} \otimes \mathcal{L}_{B}^{-1}\right)
\end{aligned}
$$

Thus, it is given by the number of fermion zero modes on the Calabi-Yau 3-fold $X$. The number of fermion zero modes on $\Sigma_{1} \cdot \Sigma_{2}$ obtained in (201) is equal to the number of zero modes obtained above :

$$
\begin{aligned}
& I_{X}\left(\left(\Sigma_{1}, E_{\Sigma_{1}}\right),\left(\Sigma_{2}, E_{\Sigma_{2}}\right)\right)=-I_{X}\left(\left(\Sigma_{2}, E_{\Sigma_{2}}\right),\left(\Sigma_{1}, E_{\Sigma_{1}}\right)\right) \\
= & \int_{X}\left(i_{\Sigma_{2}}\right)_{*}\left(\operatorname{ch}\left(E_{\Sigma_{2}}^{*} \otimes \mathcal{L}_{B}\right) \sqrt{\frac{\hat{A}\left(T \Sigma_{2}\right)}{\hat{A}\left(\mathcal{N}_{\Sigma_{2} \mid X}\right)}}\right) \wedge\left(i_{\Sigma_{1}}\right)_{*}\left(\operatorname{ch}\left(E_{\Sigma_{1}} \otimes \mathcal{L}_{B}^{-1}\right) \sqrt{\frac{\hat{A}\left(T \Sigma_{1}\right)}{\hat{A}\left(\mathcal{N}_{\Sigma_{1} \mid X}\right)}}\right) \\
= & \int_{\Sigma_{1} \cdot \Sigma_{2}}\left(\left.\left(i_{\Sigma_{1}}\right)\right|_{\Sigma_{1} \cdot \Sigma_{2}}\right)^{*}\left(\operatorname{ch}\left(E_{\Sigma_{2}}^{*} \otimes \mathcal{L}_{B}\right) \sqrt{\frac{\hat{A}\left(T \Sigma_{2}\right)}{\hat{A}\left(\mathcal{N}_{\Sigma_{2} \mid X}\right)}}\right) \wedge
\end{aligned}
$$




$$
\begin{aligned}
& \left(\left.\left(i_{\Sigma_{2}}\right)\right|_{\Sigma_{1} \cdot \Sigma_{2}}\right)^{*}\left(\operatorname{ch}\left(E_{\Sigma_{1}} \otimes \mathcal{L}_{B}^{-1}\right) \sqrt{\frac{\hat{A}\left(T \Sigma_{1}\right)}{\hat{A}\left(\mathcal{N}_{\Sigma_{1} \mid X}\right)}}\right) \\
& =\int_{\Sigma_{1} \cdot \Sigma_{2}} \operatorname{ch}\left(\left(\left.\left(i_{\Sigma_{1}}\right)\right|_{\Sigma_{1} \cdot \Sigma_{2}}\right)^{*} E_{\Sigma_{2}}^{*} \otimes\left(\left.\left(i_{\Sigma_{2}}\right)\right|_{\Sigma_{1} \cdot \Sigma_{2}}\right)^{*} E_{\Sigma_{1}}\right) \hat{A}\left(T\left(\Sigma_{1} \cdot \Sigma_{2}\right)\right) \\
& =N_{F} .
\end{aligned}
$$

The Ramond-Ramond charge of an orientifold plane ${ }^{18} W \subset X$ is given by (e.g. [56, 67])

$$
v_{X}(W)=-\left(i_{W}\right)_{*}\left[2^{\operatorname{dim}_{\mathbf{R}} W-\operatorname{dim}_{\mathbf{C}} X} \sqrt{\frac{L\left(\frac{1}{4} T W\right)}{L\left(\frac{1}{4} N_{W \mid X}\right)}}\right] .
$$

The multiplicities of chiral multiplets in the anti-symmetric and symmetric representations are given, respectively, by

$$
\begin{aligned}
\text { a.sym : } & \frac{1}{2}\left(I_{X}\left(v_{X}\left(\Sigma_{1}, E_{\Sigma_{1}}\right), v_{X}\left(\Sigma_{1^{\prime}}, E_{\Sigma_{1^{\prime}}}\right)\right)-I_{X}\left(v_{X}\left(\Sigma_{1}, E_{\Sigma_{1}}\right), v_{X}(W)\right)\right), \\
\text { sym : } & \frac{1}{2}\left(I_{X}\left(v_{X}\left(\Sigma_{1}, E_{\Sigma_{1}}\right), v_{X}\left(\Sigma_{1^{\prime}}, E_{\Sigma_{1^{\prime}}}\right)\right)+I_{X}\left(v_{X}\left(\Sigma_{1}, E_{\Sigma_{1}}\right), v_{X}(W)\right)\right) .
\end{aligned}
$$

In an application to a system of intersecting D7-branes and an O7-plane, $2^{\operatorname{dim}_{\mathbf{R}} W-\operatorname{dim}_{\mathbf{C}} X}=2$. They are also expressed by integration over the intersection curve $\Sigma_{1} \cdot \Sigma_{1^{\prime}}=\Sigma_{1} \cdot W$. Note that $\hat{A}$ and $L$ do not contribute in integration over complex curves, since they do not have 2 -form components. Thus, the multiplicities are

$$
\begin{array}{ll}
\text { a.sym. : } & \frac{1}{2}\left(\int_{\Sigma_{1} \cdot \Sigma_{1^{\prime}}}\left(\frac{F_{\Sigma_{1}}}{2 \pi}-\frac{F_{\Sigma_{1^{\prime}}}}{2 \pi}\right)+\int_{\Sigma_{1} \cdot W} 2\left(\frac{F_{\Sigma_{1}}}{2 \pi}-\frac{B}{(2 \pi)^{2} \alpha^{\prime}}\right)\right), \\
\text { sym. : } & \frac{1}{2}\left(\int_{\Sigma_{1} \cdot \Sigma_{1^{\prime}}}\left(\frac{F_{\Sigma_{1}}}{2 \pi}-\frac{F_{\Sigma_{1^{\prime}}}}{2 \pi}\right)-\int_{\Sigma_{1} \cdot W} 2\left(\frac{F_{\Sigma_{1}}}{2 \pi}-\frac{B}{(2 \pi)^{2} \alpha^{\prime}}\right)\right) .
\end{array}
$$

SInce the orientifold projection condition on the intersection curve $\Sigma_{1} \cdot \Sigma_{1^{\prime}}=\Sigma_{1} \cdot W$ is

$$
\left(\frac{F_{\Sigma_{1}}}{2 \pi}-\frac{B}{(2 \pi)^{2} \alpha^{\prime}}\right)=-\left(\frac{F_{\Sigma_{1^{\prime}}}}{2 \pi}-\frac{B}{(2 \pi)^{2} \alpha^{\prime}}\right),
$$

no chiral multiplets in the symmetric representation arise on the normal orientifold planes, and the multiplicity of the anti-symmetric representations is given by (21).

\footnotetext{
${ }^{18}$ Newly added in version 2, March, 2006.
} 


\section{References}

[1] U. Amaldi, W. de Boer and H. Furstenau, "Comparison of grand unified theories with electroweak and strong coupling constants measured at LEP," Phys. Lett. B 260 (1991) 447 ;

P. Langacker and M. x. Luo, "Implications of precision electroweak experiments for M(t), rho(0), sin**2-Theta(W) and grand unification," Phys. Rev. D 44 (1991) 817;

J. R. Ellis, S. Kelley and D. V. Nanopoulos, "Probing The Desert Using Gauge Coupling Unification," Phys. Lett. B 260 (1991) 131.

[2] N. Sakai and T. Yanagida, "Proton Decay In A Class Of Supersymmetric Grand Unified Models," Nucl. Phys. B 197, 533 (1982);

S. Weinberg, "Supersymmetry At Ordinary Energies. 1. Masses And Conservation Laws," Phys. Rev. D 26, 287 (1982).

[3] Y. Hayato et al. [Super-Kamiokande Collaboration], "Search for proton decay through $\mathrm{p} \rightarrow$ anti-nu K+ in a large water Cherenkov detector," Phys. Rev. Lett. 83 (1999) 1529 arXiv:hep-ex/9904020.

[4] T. Goto and T. Nihei, "Effect of RRRR dimension 5 operator on the proton decay in the minimal SU(5) SUGRA GUT model," Phys. Rev. D 59 (1999) 115009 arXiv:hep-ph/9808255, and references therein;

H. Murayama and A. Pierce, "Not even decoupling can save minimal supersymmetric SU(5)," Phys. Rev. D 65 (2002) 055009 arXiv:hep-ph/0108104.

[5] S. Dimopoulos and F. Wilczek, Report No. NSF-ITP-82-07.

[6] R. Barbieri, G. R. Dvali and A. Strumia, "Strings versus supersymmetric GUTs: Can they be reconciled?," Phys. Lett. B 333, 79 (1994) arXiv:hep-ph/9404278.

[7] S. M. Barr, "The stability of the gauge hierarchy in SU(5) x SU(5)," Phys. Rev. D 55, 6775 (1997) arXiv:hep-ph/9607359.

[8] E. Witten, "Deconstruction, G(2) holonomy, and doublet-triplet splitting," arXiv:hep-ph/0201018.

[9] M. Dine, Y. Nir and Y. Shadmi, "Product groups, discrete symmetries, and grand unification," Phys. Rev. D 66, 115001 (2002) arXiv:hep-ph/0206268.

[10] A. Masiero, D. V. Nanopoulos, K. Tamvakis and T. Yanagida, "Naturally Massless Higgs Doublets In Supersymmetric SU(5)," Phys. Lett. B 115 (1982) 380. 
[11] T. Yanagida, "Naturally light Higgs doublets in the supersymmetric grand unified theories with dynamical symmetry breaking," Phys. Lett. B 344, 211 (1995) arXiv:hep-ph/9409329.

[12] J. Hisano and T. Yanagida, "An N=2 SUSY Gauge Model for Dynamical Breaking of the Grand Unified SU(5) Symmetry," Mod. Phys. Lett. A 10, 3097 (1995) arXiv:hep-ph/9510277.

[13] K. I. Izawa and T. Yanagida, "R-invariant natural unification," Prog. Theor. Phys. 97, 913 (1997) arXiv:hep-ph/9703350.

[14] P. Candelas, G. T. Horowitz, A. Strominger and E. Witten, "Vacuum Configurations For Superstrings," Nucl. Phys. B 258, 46 (1985).

[15] E. Witten, "Symmetry Breaking Patterns In Superstring Models," Nucl. Phys. B 258, 75 (1985).

[16] L. J. Hall and Y. Nomura, "Gauge unification in higher dimensions," Phys. Rev. D 64 (2001) 055003 arXiv:hep-ph/0103125.

[17] Y. Imamura, T. Watari and T. Yanagida, "Semi-simple group unification in the supersymmetric brane world," Phys. Rev. D 64, 065023 (2001) arXiv:hep-ph/0103251.

[18] T. Watari and T. Yanagida, "Semi-simple unification on T(6)/Z(12) orientifold in the type IIB supergravity," Phys. Lett. B 520, 322 (2001) arXiv:hep-ph/0108057.

[19] T. Watari and T. Yanagida, "A solution to the doublet triplet splitting problem in the type IIB supergravity," arXiv:hep-ph/0208107.

[20] G. Aldazabal, L. E. Ibanez, F. Quevedo and A. M. Uranga, "D-branes at singularities: A bottom-up approach to the string embedding of the standard model," JHEP 0008, 002 (2000) arXiv:hep-th/0005067.

[21] J. R. Ellis, J. F. Gunion, H. E. Haber, L. Roszkowski and F. Zwirner, "Higgs Bosons In A Nonminimal Supersymmetric Model," Phys. Rev. D 39 (1989) 844.

[22] J. Sato and T. Yanagida, "Large lepton mixing in seesaw models: Coset-space family unification," Nucl. Phys. Proc. Suppl. 77 (1999) 293 arXiv:hep-ph/9809307.

[23] P. Ramond, "Neutrinos: A glimpse beyond the standard model," Nucl. Phys. Proc. Suppl. 77 (1999) 3 arXiv:hep-ph/9809401.

[24] L. J. Hall, H. Murayama and N. Weiner, "Neutrino mass anarchy," Phys. Rev. Lett. 84 (2000) 2572 arXiv:hep-ph/9911341. 
[25] L. E. Ibanez and G. G. Ross, "Discrete Gauge Symmetry Anomalies," Phys. Lett. B 260, 291 (1991);

T. Banks and M. Dine, "Note on discrete gauge anomalies," Phys. Rev. D 45, 1424 (1992) arXiv:hep-th/9109045;

L. E. Ibanez, "More about discrete gauge anomalies," Nucl. Phys. B 398, 301 (1993) arXiv:hep-ph/9210211.

[26] K. Kurosawa, N. Maru and T. Yanagida, "Nonanomalous R-symmetry in supersymmetric unified theories of quarks and leptons," Phys. Lett. B 512, 203 (2001) arXiv:hep-ph/0105136.

[27] M. Fujii and T. Watari, "Proton decay in the semi-simple unification," Phys. Lett. B 527, 106 (2002) arXiv:hep-ph/0112152.

[28] M. Ibe and T. Watari, "Upper bound of proton life-time in product-group unification," Phys. Rev. D 67, 114021 (2003) arXiv:hep-ph/0303123.

[29] T. Yanagida, in Proc. Workshop on the Unified Theory and the Baryon Number in the Universe, Tsukuba, 1979, eds. O. Sawada and S. Sugamoto, Report KEK-79-18 (1979); M. Gell-mann, P. Ramond and R. Slansky, in "Supergravity" North-Holland, Amsterdam, 1979, eds. D.Z. Freedman and P. van Nieuwenhuizen.

[30] D. E. Kaplan, G. D. Kribs and M. Schmaltz, "Supersymmetry breaking through transparent extra dimensions," Phys. Rev. D 62 (2000) 035010 arXiv:hep-ph/9911293;

Z. Chacko, M. A. Luty, A. E. Nelson and E. Ponton, "Gaugino mediated supersymmetry breaking," JHEP 0001 (2000) 003 arXiv:hep-ph/9911323.

[31] J. R. Ellis, D. V. Nanopoulos and K. A. Olive, "Lower limits on soft supersymmetrybreaking scalar masses," Phys. Lett. B 525 (2002) 308 arXiv:hep-ph/0109288.

[32] A. Anisimov, M. Dine, M. Graesser and S. Thomas, "Brane world SUSY breaking," Phys. Rev. D 65 (2002) 105011 arXiv:hep-th/0111235,

"Brane world SUSY breaking from string/M theory," JHEP 0203 (2002) 036 arXiv:hep-th/0201256.

[33] M. Fujii and K. Suzuki, "Investigation of noscale supersymmetry breaking models with a gauged U(1)(B-L) symmetry," Phys. Rev. D 66 (2002) 035002 arXiv:hep-ph/0202038; M. Endo, M. Yamaguchi and A. Yotsuyanagi, "Raising sfermion masses by adding extra matter fields," arXiv:hep-ph/0310043. 
[34] O. DeWolfe and S. B. Giddings, "Scales and hierarchies in warped compactifications and brane worlds," Phys. Rev. D 67 (2003) 066008 arXiv:hep-th/0208123;

M. Grana, T. W. Grimm, H. Jockers and J. Louis, "Soft supersymmetry breaking in Calabi-Yau orientifolds with D-branes and fluxes," arXiv:hep-th/0312232.

[35] K. Becker, M. Becker and A. Strominger, "Five-branes, membranes and nonperturbative string theory," Nucl. Phys. B 456, 130 (1995) arXiv:hep-th/9507158.

[36] H. Ooguri, Y. Oz and Z. Yin, "D-branes on Calabi-Yau spaces and their mirrors," Nucl. Phys. B 477, 407 (1996) arXiv:hep-th/9606112.

[37] B. S. Acharya, "On realising $\mathrm{N}=1$ super Yang-Mills in $\mathrm{M}$ theory," arXiv:hep-th/0011089;

M. Atiyah and E. Witten, "M-theory dynamics on a manifold of G(2) holonomy," Adv. Theor. Math. Phys. 6, 1 (2003) arXiv:hep-th/0107177;

B. Acharya and E. Witten, "Chiral fermions from manifolds of G(2) holonomy," arXiv:hep-th/0109152.

[38] T. Friedmann and E. Witten, "Unification scale, proton decay, and manifolds of G(2) holonomy," arXiv:hep-th/0211269.

[39] A. M. Uranga, "Local models for intersecting brane worlds," JHEP 0212, 058 (2002) arXiv:hep-th/0208014.

[40] M. R. Douglas and G. W. Moore, "D-branes, Quivers, and ALE Instantons," arXiv:hep-th/9603167;

I. Antoniadis, E. Kiritsis and J. Rizos, "Anomalous U(1)s in type I superstring vacua," Nucl. Phys. B 637 (2002) 92 arXiv:hep-th/0204153.

[41] J. Polchinski and Y. Cai, "Consistency Of Open Superstring Theories," Nucl. Phys. B 296 (1988) 91;

C. G. . Callan, C. Lovelace, C. R. Nappi and S. A. Yost, "Loop Corrections To Superstring Equations Of Motion," Nucl. Phys. B 308 (1988) 221;

E. G. Gimon and J. Polchinski, "Consistency Conditions for Orientifolds and DManifolds," Phys. Rev. D 54 (1996) 1667 arXiv:hep-th/9601038.

[42] E. G. Gimon and C. V. Johnson, "Multiple realisations of $\mathcal{N}=1$ vacua in six dimensions," Nucl. Phys. B 479 (1996) 285 arXiv:hep-th/9606176;

J. D. Blum and K. A. Intriligator, "Consistency conditions for branes at orbifold singularities," Nucl. Phys. B 506, 223 (1997) arXiv:hep-th/9705030. 
[43] J. Polchinski and A. Strominger, "New Vacua for Type II String Theory," Phys. Lett. B 388 (1996) 736 arXiv:hep-th/9510227;

J. Michelson, "Compactifications of type IIB strings to four dimensions with non-trivial classical potential," Nucl. Phys. B 495 (1997) 127 arXiv:hep-th/9610151;

G. Curio, A. Klemm, D. Lust and S. Theisen, "On the vacuum structure of type II string compactifications on Calabi-Yau spaces with H-fluxes," Nucl. Phys. B 609 (2001) 3 arXiv:hep-th/0012213;

S. B. Giddings, S. Kachru and J. Polchinski, "Hierarchies from fluxes in string compactifications," Phys. Rev. D 66 (2002) 106006 arXiv:hep-th/0105097;

S. Kachru, M. B. Schulz and S. Trivedi, "Moduli stabilization from fluxes in a simple IIB orientifold," JHEP 0310 (2003) 007 arXiv:hep-th/0201028.

[44] S. Kachru, M. B. Schulz and S. Trivedi in [43].

[45] J. A. Harvey and G. W. Moore, "On the algebras of BPS states," Commun. Math. Phys. 197 (1998) 489 arXiv:hep-th/9609017.

[46] M. Bershadsky, C. Vafa and V. Sadov, "D-Branes and Topological Field Theories," Nucl. Phys. B 463 (1996) 420 arXiv:hep-th/9511222.

[47] S. Kachru, S. Katz, A. E. Lawrence and J. McGreevy, "Open string instantons and superpotentials," Phys. Rev. D 62 (2000) 026001 arXiv:hep-th/9912151.

[48] R. Minasian and G. W. Moore, "K-theory and Ramond-Ramond charge," JHEP 9711 (1997) 002 arXiv:hep-th/9710230.

[49] E. Witten, "Five-brane effective action in M-theory," J. Geom. Phys. 22 (1997) 103 arXiv:hep-th/9610234.

[50] F. Hirzebruch, "Topological Methods in Algebraic Geometry", Springer, 1978.

[51] I. Brunner, M. R. Douglas, A. E. Lawrence and C. Romelsberger, "D-branes on the quintic," JHEP 0008 (2000) 015 arXiv:hep-th/9906200.

[52] S. Mukai, "Symplectic structure of the moduli of sheaves on an abelian or K3 surface," Invent. Math. 77 (1984) 101.

[53] M. B. Green, J. A. Harvey and G. W. Moore, "I-brane inflow and anomalous couplings on D-branes," Class. Quant. Grav. 14 (1997) 47 arXiv:hep-th/9605033.

[54] C. Romelsberger, "(Fractional) intersection numbers, tadpoles and anomalies," arXiv:hep-th/0111086. 
[55] R. Blumenhagen, V. Braun, B. Kors and D. Lust, "Orientifolds of K3 and Calabi-Yau manifolds with intersecting D-branes," JHEP 0207 (2002) 026 arXiv:hep-th/0206038.

[56] I. Brunner and K. Hori, "Orientifolds and mirror symmetry," arXiv:hep-th/0303135.

[57] L. E. Ibanez, R. Rabadan and A. M. Uranga, "Anomalous U(1)'s in type I and type IIB $\mathrm{D}=4, \mathrm{~N}=1$ string vacua," Nucl. Phys. B 542 (1999) 112 arXiv:hep-th/9808139.

[58] M. R. Douglas, "Branes within branes," arXiv:hep-th/9512077.

[59] Y. K. Cheung and Z. Yin, "Anomalies, branes, and currents," Nucl. Phys. B 517 (1998) 69 arXiv:hep-th/9710206.

[60] J. F. Morales, C. A. Scrucca and M. Serone, "Anomalous couplings for D-branes and O-planes," Nucl. Phys. B 552 (1999) 291 arXiv:hep-th/9812071;

B. J. Stefanski, "Gravitational couplings of D-branes and O-planes," Nucl. Phys. B 548 (1999) 275 arXiv:hep-th/9812088;

C. A. Scrucca and M. Serone, "Anomalies and inflow on D-branes and O-planes," Nucl. Phys. B 556 (1999) 197 arXiv:hep-th/9903145.

[61] T. Watari and T. Yanagida, "Geometric origin of large lepton mixing in a higher dimensional spacetime," Phys. Lett. B 544 (2002) 167 arXiv:hep-ph/0205090, see also T. Watari and T. Yanagida, "Higher dimensional supersymmetry as an origin of the three families for quarks and leptons," Phys. Lett. B 532 (2002) 252 arXiv:hep-ph/0201086.

[62] A. Hebecker and J. March-Russell, "The flavour hierarchy and see-saw neutrinos from bulk masses in 5d orbifold GUTs," Phys. Lett. B 541 (2002) 338 arXiv:hep-ph/0205143.

[63] Y. Fukuda et al. [Super-Kamiokande Collaboration], "Evidence for oscillation of atmospheric neutrinos," Phys. Rev. Lett. 81 (1998) 1562 arXiv:hep-ex/9807003.

[64] Y. Fukuda et al. [Super-Kamiokande Collaboration], "Measurements of the solar neutrino flux from Super-Kamiokande's first 300 days," Phys. Rev. Lett. 81 (1998) 1158 [Erratum-ibid. 81 (1998) 4279] arXiv:hep-ex/9805021,

"Constraints on neutrino oscillations using 1258 days of Super-Kamiokande solar neutrino data," Phys. Rev. Lett. 86 (2001) 5656 arXiv:hep-ex/0103033.

[65] Q. R. Ahmad et al. [SNO Collaboration], "Direct evidence for neutrino flavor transformation from neutral-current interactions in the Sudbury Neutrino Observatory," Phys. Rev. Lett. 89 (2002) 011301 arXiv:nucl-ex/0204008. 
[66] K. Eguchi et al. [KamLAND Collaboration], "First results from KamLAND: Evidence for reactor anti-neutrino disappearance," Phys. Rev. Lett. 90 (2003) 021802 arXiv:hep-ex/0212021.

[67] I. Brunner, K. Hori, K. Hosomichi and J. Walcher, "Orientifolds of Gepner models," arXiv:hep-th/0401137.

[68] D. S. Freed and E. Witten, "Anomalies in string theory with D-branes," arXiv:hep-th/9907189. 